\title{
Computation of Greeks for Asset Price Dynamics Driven by Stable and Tempered Stable Processes
}

\author{
REIICHIRO KAWAI* AND ATSUSHI TAKEUCHI ${ }^{\dagger}$
}

\begin{abstract}
The purpose of this paper is to derive the Greeks formulas of Delta, Gamma, Vega and Theta, for derivative securities with both continuous and discontinuous payoff structures under asset price dynamics described by stable and tempered stable processes with presentation of their practical simulation methods. Our approach is based on the representation of stable distributions using exponential distribution whose scaling property with respect to the Girsanov transform is used in the Malliavin calculus framework on the Poisson space. Numerical results are presented to illustrate the effectiveness of our formulas in Monte Carlo simulations relative to the finite difference method.

Keywords: CGMY process, finite difference method, Greeks, Lévy process, Malliavin calculus, Monte Carlo simulation, sensitivity analysis.

2010 Mathematics Subject Classification: 60E07, 60G51, 91B70, 60H07.
\end{abstract}

\section{Introduction}

The Brownian motion assumption on log asset price dynamics delivers convenient features to the market setting, such as market completeness, unique derivatives pricing without arbitrage and perfect dynamic hedging strategies. However, statistical analysis of log returns indicates a large deviation from Gaussian distribution, for example, through the asymmetry and the presence of excess kurtosis relative to the normal distribution. (See the pioneering papers by Mandelbrot [16] and Fama [8].) Moreover, in the risk neutral distribution for derivatives pricing, the existence of smiles in Black-Scholes implied volatilities indicates heavy tails of log returns. Amongst several candidates as a replacement of the Brownian motion suggested in the finance literature, the class of pure-jump Lévy processes has been one main streamline, since it has higher distributional flexibility and still shares the feature of stationary independent increments.

Since the work of Mandelbrot [16], the stable process has been considered by many authors for log asset price dynamics modeling. On one hand, symmetric stable processes are used for option pricing, while a drawback is the infinite call option price due to the presence of positive jumps of the stable process. On the other hand, the finite call value is achieved by imposing the maximum negative skewness on the stable process in Carr and $\mathrm{Wu}$ [4]. The log returns of those models described by stable processes violates the condition of a finite variance for the central limit theorem, while it prevents the fast vanishing of skewness of the index level.

More recently, a class of Lévy processes with exponentially tempered stable Lévy measure has attracted more attention among financial mathematicians and market practitioners. This class has appeared in the literature under several different terms, while tempered stable process seems to have been the most common so far. Tempered stable processes capture many stylized facts of log returns, such as skewness, excess kurtosis, semi-heavy tails, a stable-like behavior in short time and aggregational Gaussianity, while they preserve some mathematical tractability. The class of the tempered stable distributions is first proposed by Tweedie [20] and its associated Lévy processes are thoroughly investigated from a theoretical point of view recently by Rosiński [17]. For the use of the tempered stable distributions in financial modeling, see, for example, Carr et al. [3] and Eberlein and Madan [6].

In this paper, we derive the Greeks formulas for asset price dynamics described by stable and tempered stable processes of high practical interest. The sensitivity analysis for asset price models has been a central issue for the sake of dynamic hedging in daily practice. Fournié et al. [9] is the first to derive Greeks formulas using the integration-by-parts formula of the Malliavin calculus on the Wiener space. Our approach here is based upon the application of the Malliavin calculus on

Published in Quantitative Finance, doi:10.1080/14697688.2011.589403.

*Email Address: reiichiro.kawai@le.ac.uk. Postal Address: Department of Mathematics, University of Leicester, Leicester LE1 7RH, UK.

${ }^{\dagger}$ Email Address: takeuchi@sci.osaka-cu.ac.jp. Postal Address: Department of Mathematics, Osaka City University, Sugimoto 3-3-138, Osaka, 558-8585, Japan. 
the Poisson space of Bismut [2] to the scaling property of the exponential distribution, which appears in the well known representation of the stable random distribution with respect to the Girsanov density transform. For the tempered stable process model, we further employ the absolute continuity of the tempered stable process with respect to its associated nontempered stable process. The presentation of our Greeks formulas provides a direct help for practical use in particular of the tempered stable process setting, whose sample generation is in general not straightforward. Moreover, our study expands the applicable class of underlying Lévy processes in the context of the computation of Greeks, which has so far consisted of Poisson processes [7], compound Poisson processes [1], gamma and variance gamma processes [12, 13], Meixner processes [15], time-changed Brownian motions [10], and stochastic differential equations with jumps [19].

The rest of this paper is organized as follows. Section 2 is devoted to a review of basic facts we employ throughout this paper. First, we describe the scaling property of gamma and exponential distributions with respect to the Girsanov density transform. We next recall basic properties of the stable process, in particular, the representation of the stable marginal with the uniform and exponential distributions. In Section 3 , we derive the Greeks formulas for finite log stable and tempered stable process models. To maintain the flow of the paper, we collect proofs in Appendix. The derivation of our formulas entails rather lengthy proofs of somewhat routine nature. To avoid overloading the paper, we omit nonessential details in some instances. Section 4 presents some numerical results to illustrate the effectiveness of our formulas in the Monte Carlo estimation relative to the finite difference method. Finally, Section 5 concludes.

\section{Preliminaries}

Let us begin with general notations which will be used throughout the paper. We denote by $\mathbb{N}$ the set of positive integers. We denote by $\mathbb{R}$ be the one-dimensional Euclidean space with the norm $|\cdot|$. We write $\mathbb{R}_{+}:=(0,+\infty)$ and $\mathbb{R}_{-}:=(-\infty, 0)$. We denote by $\mathbb{E}_{\mathbb{P}}[\cdot]$ and $\operatorname{Var}_{\mathbb{P}}(\cdot)$ respectively the expectation and the variance taken under a probability measure $\mathbb{P}$. The restriction of a probability measure $\mathbb{P}$ to the $\sigma$-field $\mathscr{F}$ is denoted by $\mathbb{P} \mid \mathscr{F}$. Henceforth, we fix $(\Omega, \mathscr{F}, \mathbb{P})$ as our underlying probability space. In particular, we assume throughout the paper that $\mathbb{P}$ is a risk-neutral probability measure, which is one of infinitely many of its kind, as we consider models driven by Lévy processes. We denote by $\stackrel{\mathscr{L}}{=}$ the identity in law under a suitable probability measure. Finally, $C_{b}^{k}$ denotes the class of $k$-time continuously differentiable functions with bounded derivatives, while the subscripts $K$ of $C_{K}$ indicates the compact support and $\|\cdot\|_{\infty}$ indicates the supremum norm of continuous functions.

\subsection{Gamma and Exponential Distributions}

We here recall the definition and some key properties of the gamma and exponential distributions, which serve as important building blocks for our discussion. A random variable $Y$ is said to have a gamma distribution if its density function $f_{\mathbb{P}}$ (under the probability measure $\mathbb{P}$ ) is given in closed form by

$$
f_{\mathbb{P}}(x)=\frac{b^{a}}{\Gamma(a)} x^{a-1} e^{-b x}, \quad x \in[0,+\infty),
$$

with some $a>0$ and $b>0$. Obviously, when $a=1$, the above gamma distribution reduces to the exponential distribution with mean $1 / b$. Define the set

$$
\Lambda:=\left\{\lambda \in \mathbb{R}: \mathbb{E}_{\mathbb{P}}\left[e^{\lambda Y}\right]<+\infty\right\}=(-\infty, b),
$$

and for $\lambda \in \Lambda$, define the probability measure $\mathbb{Q}_{\lambda}$ via the so-called Esscher transform

$$
\left.\frac{d \mathbb{Q}_{\lambda}}{d \mathbb{P}}\right|_{\mathscr{G}}:=\frac{e^{\lambda Y}}{\mathbb{E}_{\mathbb{P}}\left[e^{\lambda Y}\right]}=\exp \left[\lambda Y-a \ln \frac{b}{b-\lambda}\right],
$$

where $\mathscr{G}$ is the minimal $\sigma$-field generated by $Y$. Under the probability measure $\mathbb{Q}_{\lambda}$, the random variable $Y$ again follows a gamma distribution with the density function

$$
f_{\mathbb{Q}_{\lambda}}(x)=\frac{e^{\lambda x}}{\mathbb{E}_{\mathbb{P}}\left[e^{\lambda Y}\right]} f_{\mathbb{P}}(x)=\frac{(b-\lambda)^{a}}{\Gamma(a)} x^{a-1} e^{-(b-\lambda) x}, \quad x \in[0,+\infty) .
$$

This implies that for each $z \in \mathbb{R}_{+}$,

$$
\mathbb{Q}_{\lambda}(Y \leq z)=\mathbb{P}\left(\frac{b}{b-\lambda} Y \leq z\right)
$$

(See Lemma 2.1 of Kawai and Takeuchi [12].) Throughout this paper, we call this invariance the scaling property of the gamma and exponential distributions. 


\subsection{Stable Processes}

Define independent Lévy processes $\left\{L_{-, t}: t \geq 0\right\}$ and $\left\{L_{+, t}: t \geq 0\right\}$ by characteristic functions

$$
\begin{aligned}
& \mathbb{E}_{\mathbb{P}}\left[e^{i y L_{-}, t}\right]=\exp \left[t C_{-} \Gamma\left(-\alpha_{-}\right)\left(\cos \frac{\pi \alpha_{-}}{2}\right)|y|^{\alpha_{-}}\left(1+i \tan \frac{\pi \alpha_{-}}{2} \operatorname{sgn}(y)\right)\right], \\
& \mathbb{E}_{\mathbb{P}}\left[e^{i y L_{+}, t}\right]=\exp \left[t C_{+} \Gamma\left(-\alpha_{+}\right)\left(\cos \frac{\pi \alpha_{+}}{2}\right)|y|^{\alpha_{+}}\left(1-i \tan \frac{\pi \alpha_{+}}{2} \operatorname{sgn}(y)\right)\right],
\end{aligned}
$$

with $C_{-}, C_{+} \geq 0$ and $\alpha_{-}, \alpha_{+} \in(0,1) \cup(1,2)$. Both $\left\{L_{-, t}: t \geq 0\right\}$ and $\left\{L_{+, t}: t \geq 0\right\}$ are stable processes without Gaussian component and with Lévy measures $v_{(\mathrm{s},-)}$ and $v_{(\mathrm{s},+)}$ given respectively by

$$
v_{(\mathrm{s},-)}(d z)=C_{-} \frac{d z}{|z|^{1+\alpha_{-}}} \mathbb{1}_{\mathbb{R}_{-}}(z), \quad v_{(\mathrm{s},+)}(d z)=C_{+} \frac{d z}{z^{1+\alpha_{+}}} \mathbb{1}_{\mathbb{R}_{+}}(z) .
$$

If $\alpha_{-}$and $\alpha_{+}$are smaller than 1 , then they are subordinators respectively in the negative and positive directions. When $\alpha_{-}$and $\alpha_{+}$are greater than 1 , they are centered with jumps only in a single direction. Moreover, define the superposition

$$
\left\{L_{t}: t \geq 0\right\}:=\left\{L_{-, t}+L_{+, t}: t \geq 0\right\},
$$

which is also a stable process only if $\alpha_{-}=\alpha_{+}$.

Recall that for each $t>0, \mathbb{E}\left[\left|L_{-, t}\right|^{\theta}\right]$ is finite if $\theta \in\left(0, \alpha_{-}\right)$, while is infinite if $\theta \geq \alpha_{-}$. Moreover, for $\alpha_{-} \in(0,1) \cup$ $(1,2)$, as $x \downarrow-\infty$,

$$
\mathbb{P}\left(L_{-, t}<x\right) \sim \frac{t C_{-}}{\alpha_{-}}|x|^{-\alpha_{-}},
$$

while the right tail simply does not exist if $\alpha_{-} \in(0,1)$, or is extremely light if $\alpha_{-} \in(1,2)$, as $x \uparrow+\infty$,

$$
\mathbb{P}\left(L_{-, t}>x\right) \sim \frac{1}{\sqrt{2 \pi \alpha_{-}\left(\alpha_{-}-1\right)}}\left(\frac{x}{c \alpha_{-}}\right)^{-\frac{\alpha_{-}}{2\left(\alpha_{-}-1\right)}} \exp \left[-\left(\alpha_{-}-1\right)\left(\frac{x}{c \alpha_{-}}\right)^{\frac{\alpha_{-}}{\alpha_{-}-1}}\right]
$$

with a suitable constant $c$ depending on $\alpha_{-}$and $t C_{-}$. Similar results hold for the positive component $\left\{L_{+, t}: t \geq 0\right\}$ in the opposite direction of tails, as a matter of course. (See Zolotarev [21] for more details on the stable distribution.)

The stable distribution can be characterized by four parameters, often written as $S_{\alpha}(\sigma, \beta, \gamma)$, with $\sigma>0, \beta \in[-1,+1]$ and $\gamma \in \mathbb{R}$. By using the scaling property of the stable distribution $S_{\alpha}(\sigma, \beta, 0) \stackrel{\mathscr{L}}{=} \sigma S_{\alpha}(1, \beta, 0)$ for $\sigma>0$, it holds that for each $t>0$,

$$
\begin{aligned}
& L_{-, t} \stackrel{\mathscr{L}}{=} t^{\frac{1}{\alpha_{-}}} \sigma_{-} S_{\alpha_{-}}(1,-1,0), \\
& L_{+, t} \stackrel{\mathscr{L}}{=} t^{\frac{1}{\alpha_{+}}} \sigma_{+} S_{\alpha_{+}}(1,+1,0),
\end{aligned}
$$

where

$$
\begin{aligned}
& \sigma_{-}:=-C_{-} \Gamma\left(-\alpha_{-}\right) \cos \left(\pi \alpha_{-} / 2\right)>0, \\
& \sigma_{+}:=-C_{+} \Gamma\left(-\alpha_{+}\right) \cos \left(\pi \alpha_{+} / 2\right)>0 .
\end{aligned}
$$

It is worthwhile to note that if $\alpha_{-}=\alpha_{+}=: \alpha$, then it holds that $L_{t} \stackrel{\mathscr{L}}{=} S_{\alpha}(\sigma, \beta, 0)$ where $\sigma=-t\left(C_{-}+C_{+}\right) \Gamma(-\alpha) \cos (\pi \alpha / 2)$ and $\beta=\left(C_{+}-C_{-}\right) /\left(C_{+}+C_{-}\right)$. The marginals $L_{-, t}$ and $L_{+, t}$ can be simulated in the exact sense through the well known identity in law; for each $t>0$,

$$
\begin{aligned}
& L_{-, t} \stackrel{\mathscr{L}}{=} t^{\frac{1}{\alpha_{-}}} U_{-, \alpha_{-}} E_{-}^{-\frac{1-\alpha_{-}}{\alpha_{-}}}=: \widetilde{L}_{-, t}, \\
& L_{+, t} \stackrel{\mathscr{L}}{=} t^{\frac{1}{\alpha_{+}}} U_{+, \alpha_{+}} E_{+}^{-\frac{1-\alpha_{+}}{\alpha_{+}}}=: \widetilde{L}_{+, t} .
\end{aligned}
$$

Here, $E_{-}$and $E_{+}$are independent standard exponential random variables and

$$
\begin{aligned}
U_{-, \alpha_{-}} & :=\sigma_{-} \frac{\sin \left(\alpha_{-}\left(V_{-}+\theta_{-}\right)\right)}{\left(\cos \left(\alpha_{-} \theta_{-}\right) \cos V_{-}\right)^{1 / \alpha_{-}}}\left[\cos \left(\left(1-\alpha_{-}\right) V_{-}-\alpha_{-} \theta_{-}\right)\right]^{\frac{1-\alpha_{-}}{\alpha_{-}}}, \\
U_{+, \alpha_{+}} & :=\sigma_{+} \frac{\sin \left(\alpha_{+}\left(V_{+}+\theta_{+}\right)\right)}{\left(\cos \left(\alpha_{+} \theta_{+}\right) \cos V_{+}\right)^{1 / \alpha_{+}}}\left[\cos \left(\left(1-\alpha_{+}\right) V_{+}-\alpha_{+} \theta_{+}\right)\right]^{\frac{1-\alpha_{+}}{\alpha_{+}}},
\end{aligned}
$$


where $\theta_{-}=\arctan \left(-\tan \left(\pi \alpha_{-} / 2\right)\right) / \alpha_{-}, \theta_{+}=\arctan \left(\tan \left(\pi \alpha_{+} / 2\right)\right) / \alpha_{+}, V_{-}$and $V_{+}$are independent uniform random variables on $(-\pi / 2, \pi / 2)$, also independent of $E_{-}$and $E_{+}$. (See Chambers et al. [5].) For later use, we define for each $t>0$,

$$
\widetilde{L}_{t}:=\widetilde{L}_{-, t}+\widetilde{L}_{+, t}
$$

\section{Computation of Greeks}

In this section, we present Greeks formulas for European payoffs under asset price dynamics described by stable and tempered stable processes. As proved in Kawai and Takeuchi [12], the payoff function does not have to be smooth in full. More precisely, payoff functions have to be only in the class $\mathscr{J}$ defined by

$$
\mathscr{J}\left(\mathbb{R}_{+} ; \mathbb{R}\right):=\left\{f: \mathbb{R}_{+} \rightarrow \mathbb{R} \mid f=\sum_{k=1}^{n} c_{k} f_{k} \mathbb{1}_{A_{k}}, n \in \mathbb{N}, c_{k} \in \mathbb{R}, f_{k} \in C_{L}\left(\mathbb{R}_{+} ; \mathbb{R}\right), A_{k} \text { intervals on } \mathbb{R}_{+}\right\},
$$

where

$$
C_{L}\left(\mathbb{R}_{+} ; \mathbb{R}\right):=\left\{f \in C\left(\mathbb{R}_{+} ; \mathbb{R}\right)|| f(x) \mid \leq C(1+|x|) \text { for some } C>0\right\},
$$

with an additional assumption to vanish on a suitable neighborhood, which will be specified later. To maintain the flow of the paper, we collect all proofs in Appendix. Recall that $\mathbb{P}$ is a risk-neutral probability.

\subsection{Greeks for the Finite Moment Log Stable Model}

Here, we derive Greeks formulas for the finite moment log stable (FMLS) model of Carr and Wu [4], defined by

$$
S_{t}^{(\mathrm{s})}:=S_{0} e^{(r-q) t} \frac{e^{\sigma L_{-, t}}}{\mathbb{E}_{\mathbb{P}}\left[e^{\left.\sigma L_{-, t}\right]}\right.}=S_{0} e^{\left(r-q-c_{1}(\sigma)\right) t+\sigma L_{-, t}} .
$$

Here, $\sigma>0, r \geq 0, q \geq 0, \alpha_{-} \in(1,2)$,

$$
c_{1}(\sigma):=\frac{\sigma^{\alpha_{-}}}{\cos \left(\frac{\pi \alpha_{-}}{2}\right)},
$$

and $\left\{L_{-, t}: t \geq 0\right\}$ is a stable process defined by (2.4) with $C_{-}=\left(\Gamma\left(-\alpha_{-}\right) \cos \left(\pi \alpha_{-} / 2\right)\right)^{-1 / \alpha_{-}}$. The log return $\ln \left(S_{t_{2}}^{(\mathrm{s})} / S_{t_{1}}^{(\mathrm{s})}\right)$, $t_{2}>t_{1}$, has an $\alpha_{-}$-stable distribution with the tail behaviors (2.7) and (2.8), and thus has an infinite variance. However, the asset price $S_{t}^{(\mathrm{s})}$ at each fixed time has finite moments of every polynomial order thanks to the very light right tail (2.8). This implies that the asset price process 3.1 is a martingale with respect to the natural filtration generated by itself. As mentioned earlier, an asset price process cannot be a martingale as soon as its log return includes a stable random variable with positive jumps. To present our Greeks formulas, let us define for each $t>0$,

$$
\widetilde{S}_{t}^{(\mathrm{s})}:=S_{0} e^{\left(r-q-c_{1}(\sigma)\right) t+\sigma \widetilde{L}_{-, t}} \stackrel{\mathscr{L}}{=} S_{t}^{(\mathrm{s})},
$$

where $\widetilde{L}_{-, t}$ is defined by (2.9).

Theorem 3.1. Let $T>0$ and $\alpha_{-} \in(1,2)$. Let $\Phi$ be a measurable function in $\mathscr{J}\left(\mathbb{R}_{+} ; \mathbb{R}\right)$ satisfying $\Phi(x)=0$ on a neighborhood of $S_{0} e^{\left(r-q-c_{1}(\sigma)\right) T}$. Then, we have the following;

(i) Delta:

$$
\frac{\partial}{\partial S_{0}} \mathbb{E}_{\mathbb{P}}\left[\Phi\left(S_{T}^{(\mathrm{s})}\right)\right]=\frac{1}{S_{0}} \mathbb{E}_{\mathbb{P}}\left[\Phi\left(\widetilde{S}_{T}^{(\mathrm{s})}\right) \frac{1-\alpha_{-} E_{-}}{\sigma\left(1-\alpha_{-}\right) \widetilde{L}_{-, T}}\right]
$$

(ii) Gamma:

$$
\frac{\partial^{2}}{\partial S_{0}^{2}} \mathbb{E}_{\mathbb{P}}\left[\Phi\left(S_{T}^{(\mathrm{s})}\right)\right]=\frac{1}{S_{0}^{2}} \mathbb{E}_{\mathbb{P}}\left[\Phi\left(\widetilde{S}_{T}^{(\mathrm{s})}\right) \frac{\left(\alpha_{-} E_{-}-2\right)\left(\alpha_{-} E_{-}-1\right)-\alpha_{-}}{\sigma^{2}\left(1-\alpha_{-}\right)^{2} \widetilde{L}_{-, T}^{2}}\right]-\frac{1}{S_{0}} \frac{\partial}{\partial S_{0}} \mathbb{E}_{\mathbb{P}}\left[\Phi\left(S_{T}^{(\mathrm{s})}\right)\right] .
$$

(iii) Vega:

$$
\frac{\partial}{\partial \sigma} \mathbb{E}_{\mathbb{P}}\left[\Phi\left(S_{T}^{(\mathrm{s})}\right)\right]=\mathbb{E}_{\mathbb{P}}\left[\Phi\left(\widetilde{S}_{T}^{(\mathrm{s})}\right) \frac{\alpha_{-}\left(1-E_{-}\right)}{\sigma\left(1-\alpha_{-}\right)}\right]-\frac{\alpha_{-} c_{1}(\sigma) T}{\sigma} S_{0} \frac{\partial}{\partial S_{0}} \mathbb{E}_{\mathbb{P}}\left[\Phi\left(S_{T}^{(\mathrm{s})}\right)\right]
$$

(iv) Theta:

$$
\frac{\partial}{\partial T} \mathbb{E}_{\mathbb{P}}\left[\Phi\left(S_{T}^{(\mathrm{s})}\right)\right]=\mathbb{E}_{\mathbb{P}}\left[\Phi\left(\widetilde{S}_{T}^{(\mathrm{s})}\right) \frac{1-E_{-}}{\left(1-\alpha_{-}\right) T}\right]+\left(r-q-c_{1}(\sigma)\right) S_{0} \frac{\partial}{\partial S_{0}} \mathbb{E}_{\mathbb{P}}\left[\Phi\left(S_{T}^{(\mathrm{s})}\right)\right]
$$


Proof. See Appendix.

Remark 3.2. In our Greeks formulas, the random variables $\widetilde{S}_{T}^{(\mathrm{s})}$ and $\widetilde{L}_{-, T}$ cannot be replaced respectively with $S_{T}^{(\mathrm{s})}$ and $L_{-, T}$ due to the presence of the exponential random variable $E_{-}$, which forms $\widetilde{L}_{-, T}$. This would not be a serious drawback since stable random variables are most often simulated through representations (2.9) and (2.10).

Remark 3.3. The condition on the payoff function $\Phi(x)$, vanishing around $x_{0}:=S_{0} e^{\left(r-q-c_{1}(\sigma)\right) T}$, is required to avoid the explosion of the random variable $\widetilde{L}_{-, T}$ in the denominator of the Delta and Gamma formulas, since $\mathbb{E}_{\mathbb{P}}\left[\left|L_{-, T}\right|^{p}\right]=+\infty$ for $p \leq-1$ when $\alpha_{-} \in(1,2)$. Even under this condition, our formulas are still valid, for example, for (digital) call options with strike $K>x_{0}$ and (digital) put options with $K<x_{0}$.

Remark 3.4. With $\alpha_{-} \in(0,1)$, the dynamics $(3.1)$ is still a martingale with finite moments of every polynomial order. Moreover, $L_{-, T}$ has finite moments of every negative order. (See Example 25.10 of Sato [18].) Hence, the assumption imposed on $\Phi$ to vanish around $x_{0}:=S_{0} e^{\left(r-q-c_{1}(\sigma)\right) T}$ is no longer required when $\alpha_{-} \in(0,1)$. However, since then the underlying stable process $\left\{L_{-, t}: t \geq 0\right\}$ is a subordinator (in the negative direction), the dynamics is not appropriate at all as an asset price model due to the existence of its upper bound $x_{0}$.

Remark 3.5. By setting $\alpha_{-}=2$ in the model (3.1), we recover the Black-Scholes model. Moreover, the simulation method (2.9) is also valid for the case $\alpha_{-}=2$, where $\widetilde{L}_{-, t}$ is then symmetric and centered. Therefore, the above formulas holds even when $\alpha_{-}=2$ as Black-Scholes Greeks formulas. They are interesting from a theoretical point of view in the sense that they are obtained through Malliavin calculus on the Poisson space, while not quite of practical interest due to the additional condition of the vanishing payoff function, which is not required for the Greeks formulas obtained through the Malliavin calculus on the Wiener space.

Remark 3.6. We have left the theta formula as is above for simplicity in notation, while in practice, the payoff should be discounted and thus

$$
\frac{\partial}{\partial T} \mathbb{E}_{\mathbb{P}}\left[e^{-r T} \Phi\left(S_{T}^{(\mathrm{s})}\right)\right]=-r e^{-r T} \mathbb{E}_{\mathbb{P}}\left[\Phi\left(S_{T}^{(\mathrm{s})}\right)\right]+e^{-r T} \frac{\partial}{\partial T} \mathbb{E}_{\mathbb{P}}\left[\Phi\left(S_{T}^{(\mathrm{s})}\right)\right],
$$

where the first term can immediately be estimated since independent replications of the payoff are available.

\subsection{Greeks for Models with Tempered Stable Processes}

In our context, a tempered stable process $\left\{X_{t}^{(\mathrm{ts})}: t \geq 0\right\}$ is a Lévy process without Gaussian component and can be divided into two independent Lévy processes

$$
\left\{X_{t}^{(\mathrm{ts})}: t \geq 0\right\}=\left\{X_{-, t}^{(\mathrm{ts})}+X_{+, t}^{(\mathrm{ts})}: t \geq 0\right\}
$$

with characteristic functions

$$
\begin{aligned}
& \mathbb{E}_{\mathbb{P}}\left[e^{i y X_{-, t}^{(\mathrm{ts})}}\right]=\exp \left[t C_{-} \Gamma\left(-\alpha_{-}\right)\left((G+i y)^{\alpha_{-}}-G^{-\alpha_{-}}-i y \alpha_{-} G^{-1+\alpha_{-}} \mathbb{1}_{(1,2)}\left(\alpha_{-}\right)\right)\right], \\
& \mathbb{E}_{\mathbb{P}}\left[e^{i y X_{+, t}^{(\mathrm{ts})}}\right]=\exp \left[t C_{+} \Gamma\left(-\alpha_{+}\right)\left((M-i y)^{\alpha_{+}}-M^{\alpha_{+}}+i y \alpha_{+} M^{-1+\alpha_{+}} \mathbb{1}_{(1,2)}\left(\alpha_{+}\right)\right)\right],
\end{aligned}
$$

where $C_{-}, C_{+} \geq 0, G>0, M>0, \alpha_{-}, \alpha_{+} \in(0,1) \cup(1,2)$, and with Lévy measures $v_{(\mathrm{ts},-)}$ and $v_{(\mathrm{ts},+)}$ given respectively by

$$
v_{(\mathrm{ts},-)}(d z)=C_{-} \frac{e^{-G|z|}}{|z|^{1+\alpha_{-}}} \mathbb{1}_{\mathbb{R}_{-}}(z) d z, \quad v_{(\mathrm{ts},+)}(d z)=C_{+} \frac{e^{-M z}}{z^{1+\alpha_{+}}} \mathbb{1}_{\mathbb{R}_{+}}(z) d z
$$

The Lévy processes $\left\{X_{-, t}^{(\mathrm{ts})}: t \geq 0\right\}$ and $\left\{X_{+, t}^{(\mathrm{ts})}: t \geq 0\right\}$ consist of jumps only in a single direction. This accounts respectively for the downward and upward moves of the market. They are subordinators without drift (respectively in the negative and positive directions) when $\alpha_{-}$and $\alpha_{+}$are smaller than 1, while they are centered on their own when $\alpha_{-}$and $\alpha_{+}$are greater than 1 .

Consider the asset price dynamics $\left\{S_{t}^{(\mathrm{ts})}: t \geq 0\right\}$ defined by

$$
S_{t}^{(\mathrm{ts})}:=S_{0} e^{(r-q) t} \frac{e^{\sigma X_{t}^{(\mathrm{ts})}}}{\mathbb{E}_{\mathbb{P}}\left[e^{\sigma X_{t}^{(\mathrm{ts})}}\right]}=S_{0} e^{\left(r-q-c_{2}(\sigma)\right) t+\sigma X_{t}^{(\mathrm{ts})}},
$$


where $\sigma>0, M>\sigma$, and

$$
\begin{aligned}
c_{2}(\sigma):=\ln \mathbb{E}_{\mathbb{P}}\left[e^{\left.\sigma X_{1}^{(\mathrm{ts})}\right]=}\right. & C_{-} \Gamma\left(-\alpha_{-}\right)\left[(G+\sigma)^{\alpha_{-}}-G^{\alpha_{-}}-\sigma \alpha_{-} G^{-1+\alpha_{-}} \mathbb{1}_{(1,2)}\left(\alpha_{-}\right)\right] \\
& +C_{+} \Gamma\left(-\alpha_{+}\right)\left[(M-\sigma)^{\alpha_{+}}-M^{\alpha_{+}}+\sigma \alpha_{+} M^{-1+\alpha_{+}} \mathbb{1}_{(1,2)}\left(\alpha_{+}\right)\right] .
\end{aligned}
$$

This model setting is broad enough to cover many models proposed in the literature based on tempered stable processes. (See, for example, Kawai [14] for some calibration results.) The Lévy process used for asset price modeling in Carr et al. [3] is named the CGMY process after the four authors with $C_{-}=C_{+}>0$ and $\alpha_{-}=\alpha_{+}<2$. The model proposed in Eberlein and Madan [6] focuses on the case of no positive jumps by $C_{+}=0$ to describe the possible absence of short positions with longer horizon.

Let $\mathbb{Q}_{0}$ be a probability measure under which $\left\{L_{-, t}: t \geq 0\right\}$ and $\left\{L_{+, t}: t \geq 0\right\}$ are independent stable processes, respectively defined by (2.4) and (2.5), where the expectations are understood here to be taken under the probability measure $\mathbb{Q}_{0}$ instead, while $\mathbb{P}$ is the probability measure under which $\left\{X_{-, t}^{(\mathrm{ts})}: t \geq 0\right\}$ and $\left\{X_{+, t}^{(\mathrm{ts})}: t \geq 0\right\}$ are independent Lévy processes, respectively defined by (3.2) and (3.3). Let $\left(\mathscr{F}_{t}\right)_{t \geq 0}$ be the natural filtration generated by $\left\{L_{-, t}: t \geq 0\right\}$ and $\left\{L_{+, t}: t \geq 0\right\}$. It then follows from Theorem 33.2 of Sato [18] that for each $T>0$, the two probability measures $\mathbb{P} \mid \mathscr{F}_{T}$ and $\left.\mathbb{Q}_{0}\right|_{\mathscr{F}_{T}}$ are mutually absolutely continuous through the Radon-Nikodým derivative

$$
\left.\frac{d \mathbb{P}}{d \mathbb{Q}_{0}}\right|_{\mathscr{F}_{T}}=\frac{e^{G L_{-, T}-M L_{+, T}}}{\mathbb{E}_{\mathbb{Q}_{0}}\left[e^{G L_{-, T}-M L_{+}, T}\right]}=e^{G L_{-, T}-M L_{+, T}-T \gamma_{1}}, \quad \mathbb{Q}_{0}-\text { a.s. }
$$

where

$$
\gamma_{1}:=C_{-} \Gamma\left(-\alpha_{-}\right) G^{\alpha_{-}}+C_{+} \Gamma\left(-\alpha_{+}\right) M^{\alpha_{+}}
$$

Moreover, it holds that

$$
\mathbb{Q}_{0}\left(\left\{L_{t}+t \gamma_{2}: t \in[0, T]\right\} \in B\right)=\mathbb{P}\left(\left\{X_{t}^{(\mathrm{ts})}: t \in[0, T]\right\} \in B\right), \quad B \in \mathscr{B}(\mathbb{D}[0, T]) .
$$

where

$$
\gamma_{2}:=C_{-} \Gamma\left(1-\alpha_{-}\right) G^{-1+\alpha_{-}} \mathbb{1}_{(1,2)}\left(\alpha_{-}\right)-C_{+} \Gamma\left(1-\alpha_{+}\right) M^{-1+\alpha_{+}} \mathbb{1}_{(1,2)}\left(\alpha_{+}\right) .
$$

Also define, for each $t>0$, a random variable

$$
\widetilde{S}_{t}^{(\mathrm{ts})}=S_{0} e^{(r-q) t} \exp \left[\sigma\left(\widetilde{L}_{t}+t \gamma_{2}\right)-t c_{2}(\sigma)\right]
$$

Note that $\widetilde{S}_{t}^{\text {(ts) }} \stackrel{\mathscr{L}}{\neq} S_{t}^{(\mathrm{ts})}$. To simplify the presentation, we formally employ the notation

$$
\left.\frac{d \mathbb{P}}{d \mathbb{Q}_{0}}\right|_{\mathscr{G}_{1}}:=\frac{e^{G \widetilde{L}_{-, T}-M \widetilde{L}_{+, T}}}{\mathbb{E}_{\mathbb{Q}_{0}}\left[e^{G L_{-, T}-M L_{+}, T}\right]}=e^{G \widetilde{L}_{-, T}-M \widetilde{L}_{+, T}-T \gamma_{1}}, \quad \mathbb{Q}_{0}-\text { a.s. }
$$

where $\mathscr{G}_{1}$ is the minimal $\sigma$-field generated by the random variables $\widetilde{L}_{-, T}$ and $\widetilde{L}_{+, T}$. To simplify the notation, we write

$$
\begin{gathered}
J_{T}:=\eta_{-}\left(1-E_{-}-\beta_{-} G \widetilde{L}_{-, T}\right)+\eta_{+}\left(1-E_{+}+\beta_{+} M \widetilde{L}_{+, T}\right), \\
K_{T}:=\eta_{-} \beta_{-} \widetilde{L}_{-, T}+\eta_{+} \beta_{+} \widetilde{L}_{+, T}, \quad N_{T}:=\eta_{-}^{2} \beta_{-}^{2} \widetilde{L}_{-, T}+\eta_{+}^{2} \beta_{+}^{2} \widetilde{L}_{+, T},
\end{gathered}
$$

where $\beta_{-}:=\left(1-\alpha_{-}\right) / \alpha_{-}$and $\beta_{+}:=\left(1-\alpha_{+}\right) / \alpha_{+}$.

Theorem 3.7. Let $T>0$, let $\eta_{-} \in[0,1]$ and $\eta_{+} \in[0,1]$ such that $\eta_{-}+\eta_{+}>0$ and let $\Phi$ be a measurable function in $\mathscr{J}\left(\mathbb{R}_{+} ; \mathbb{R}\right)$ satisfying $\Phi(x)=0$ on a neighborhood of $S_{0} e^{\left(r-q+\gamma_{2}-c_{2}(\sigma)\right) T}$. Then, we have the following;

(i) Delta:

$$
\frac{\partial}{\partial S_{0}} \mathbb{E}_{\mathbb{P}}\left[\Phi\left(S_{T}^{(\mathrm{ts})}\right)\right]=\frac{1}{S_{0} \sigma} \mathbb{E}_{\mathbb{Q}_{0}}\left[\left.\frac{d \mathbb{P}}{d \mathbb{Q}_{0}}\right|_{\mathscr{G}_{1}} \Phi\left(\widetilde{S}_{T}^{(\mathrm{ts})}\right)\left(\frac{J_{T}}{K_{T}}+\frac{N_{T}}{K_{T}^{2}}\right)\right] .
$$

(ii) Gamma:

$$
\begin{aligned}
& \frac{\partial^{2}}{\partial S_{0}^{2}} \mathbb{E}_{\mathbb{P}}\left[\Phi\left(S_{T}^{(\mathrm{ts})}\right)\right]=\frac{1}{S_{0}^{2} \sigma^{2}} \mathbb{E}_{\mathbb{Q}_{0}}\left[\frac { d \mathbb { P } } { d \mathbb { Q } _ { 0 } } | _ { \mathscr { G } _ { 1 } } \Phi ( \widetilde { S } _ { T } ^ { ( \mathrm { ts } ) } ) \left(\frac{J_{T}^{2}+\eta_{-}^{2}\left(-E_{-}+\beta_{-}^{2} G \widetilde{L}_{-, T}\right)-\eta_{+}^{2}\left(E_{+}+\beta_{+}^{2} M \widetilde{L}_{+, T}\right)}{K_{T}^{2}}\right.\right. \\
& \left.\left.+\frac{3 N_{T} J_{T}-\eta_{-}^{3} \beta_{-}^{3} \widetilde{L}_{-, T}-\eta_{+}^{3} \beta_{+}^{3} \widetilde{L}_{+, T}}{K_{T}^{3}}+\frac{3 N_{T}^{2}}{K_{T}^{4}}\right)\right]-\frac{1}{S_{0}} \frac{\partial}{\partial S_{0}} \mathbb{E}_{\mathbb{P}}\left[\Phi\left(S_{T}^{(\mathrm{ts})}\right)\right] .
\end{aligned}
$$


(iii) Vega:

$$
\begin{array}{r}
\frac{\partial}{\partial \sigma} \mathbb{E}_{\mathbb{P}}\left[\Phi\left(S_{T}^{(\mathrm{ts})}\right)\right]=\frac{1}{\sigma} \mathbb{E}_{\mathbb{Q}_{0}}\left[\left.\frac{d \mathbb{P}}{d \mathbb{Q}_{0}}\right|_{\mathscr{G}_{1}} \Phi\left(\widetilde{S}_{T}^{(\mathrm{ts})}\right)\left(-G \widetilde{L}_{-, T}+M \widetilde{L}_{+, T}-\frac{\alpha_{-}\left(E_{-}-1\right)}{1-\alpha_{-}}-\frac{\alpha_{+}\left(E_{+}-1\right)}{1-\alpha_{+}}\right)\right] \\
+\left(\gamma_{2}-c_{2}^{\prime}(\sigma)\right) T S_{0} \frac{\partial}{\partial S_{0}} \mathbb{E}_{\mathbb{P}}\left[\Phi\left(S_{T}^{(\mathrm{ts})}\right)\right] .
\end{array}
$$

(iv) Theta:

$$
\begin{aligned}
\frac{\partial}{\partial T} \mathbb{E}_{\mathbb{P}}\left[\Phi\left(S_{T}^{(\mathrm{ts})}\right)\right]=-\gamma_{1} \mathbb{E}_{\mathbb{P}}\left[\Phi\left(S_{T}^{(\mathrm{ts})}\right)\right]+\left(r-q+\sigma \gamma_{2}-c_{2}(\sigma)\right) S_{0} \frac{\partial}{\partial S_{0}} \mathbb{E}_{\mathbb{P}}\left[\Phi\left(S_{T}^{(\mathrm{ts})}\right)\right] \\
-\frac{1}{T} \mathbb{E}_{\mathbb{Q}_{0}}\left[\left.\frac{d \mathbb{P}}{d \mathbb{Q}_{0}}\right|_{\mathscr{G}_{1}} \Phi\left(\widetilde{S}_{T}^{(\mathrm{ts})}\right)\left(\frac{E_{-}-1}{1-\alpha_{-}}+\frac{E_{+}-1}{1-\alpha_{+}}\right)\right] .
\end{aligned}
$$

Proof. See Appendix.

All our formulas in Theorem 3.1 and Theorem 3.7 require no condition on the time length $T$. This is because the time length $T$ can be extracted as a constant from the random variable $\widetilde{L}_{-, T}$ thanks to the scaling property of the stable distribution, just as in the Gaussian setting. This supports our results from a practical point of view since they can be used for dynamic hedging by choosing the time length $T$ arbitrarily small.

The Delta and Gamma formulas may look as though they had $\widetilde{L}_{-, T}$ and $\widetilde{L}_{+, T}$ in denominator, respectively, of polynomial order 2 and 4 . They are however effectively of order 1 and 2, as just should be as in the formulas of Theorem 3.1 , since the nominators cancel out their denominator in part when $\left|\widetilde{L}_{-, T}\right|+\left|\widetilde{L}_{+, T}\right| \downarrow 0$.

Remark 3.8. As discussed in Remark 3.2 , the random variables $\widetilde{S}_{T}^{\mathrm{ts})}, \widetilde{L}_{-, T}$ and $\widetilde{L}_{+, T}$ cannot be replaced respectively with $S_{T}^{(\mathrm{ts})}, L_{-, T}$ and $L_{+, T}$ due to the presence of the exponential random variables $E_{-}$and $E_{+}$. In fact, the presentation of our Greeks formulas provides a direct help for practical use since the generation of stable random variables with the associated Esscher transform is most likely the only practical simulation method for tempered stable random variables considering computing budget.

Remark 3.9. In the above Delta and Gamma formulas, the choice of the parameters $\left(\eta_{-}, \eta_{+}\right)$are arbitrary within the condition. (In fact, the ratio $\eta_{-} / \eta_{+}$only matters.) A typical criterion is the estimator variance with a view towards Monte Carlo simulation. It however seems difficult to find its a priori global minimum. We will later illustrate this point through numerical results.

Remark 3.10. We have fully made use of the absolute continuity of the tempered stable distribution with respect to its corresponding "non-tempered" stable distribution to validate the asset price dynamics (3.8). This dynamics is driven by stable processes and is almost surely finite in theory. However, extra care should be taken when implementing our results. Although the Radon-Nikodým derivative (3.9) is multiplied to the payoff in the end, the asset price dynamics may often explode itself on computer basis due to the stable random variable $\widetilde{L}_{+, T}$ with jumps in the positive direction. To avoid the explosion, it is a valid route to suppress the density transform of the positive component by $\eta_{+}=0$ and directly simulate $X_{+, T}^{\text {(ts) }}$. When $\alpha_{+} \in(0,1)$, an exact simulation methods is available through acceptance-rejection sampling. If $\alpha_{+} \in(1,2)$, we need some approximative methods since exact simulation is in general difficult. (See Kawai and Masuda [11] for various simulation methods of tempered stable random variates.)

As described earlier, we recover the CGMY model of [3] by setting $C_{-}=C_{+}$and $\alpha_{-}=\alpha_{+}$, and our formulas simplifies accordingly. The following is another obvious yet worthwhile connection between Theorem 3.1 and 3.7

Corollary 3.11. Set $\alpha_{-} \in(1,2), C_{-}=\left(\Gamma\left(-\alpha_{-}\right) \cos \left(\pi \alpha_{-} / 2\right)\right)^{-1 / \alpha_{-}}, C_{+}=0$ and $G=M=0$ in the formulas of Theorem 3.7 Then, we recover the respective formulas of Theorem 3.1.

$$
\begin{array}{cc}
\frac{\partial}{\partial S_{0}} \mathbb{E}_{\mathbb{P}}\left[\Phi\left(S_{T}^{(\mathrm{ts})}\right)\right]=\frac{\partial}{\partial S_{0}} \mathbb{E}_{\mathbb{P}}\left[\Phi\left(S_{T}^{(\mathrm{s})}\right)\right], & \frac{\partial^{2}}{\partial S_{0}^{2}} \mathbb{E}_{\mathbb{P}}\left[\Phi\left(S_{T}^{(\mathrm{ts})}\right)\right]=\frac{\partial^{2}}{\partial S_{0}^{2}} \mathbb{E}_{\mathbb{P}}\left[\Phi\left(S_{T}^{(\mathrm{s})}\right)\right], \\
\frac{\partial}{\partial \sigma} \mathbb{E}_{\mathbb{P}}\left[\Phi\left(S_{T}^{(\mathrm{ts})}\right)\right]=\frac{\partial}{\partial \sigma} \mathbb{E}_{\mathbb{P}}\left[\Phi\left(S_{T}^{(\mathrm{s})}\right)\right], & \frac{\partial}{\partial T} \mathbb{E}_{\mathbb{P}}\left[\Phi\left(S_{T}^{(\mathrm{ts})}\right)\right]=\frac{\partial}{\partial T} \mathbb{E}_{\mathbb{P}}\left[\Phi\left(S_{T}^{(\mathrm{s})}\right)\right] .
\end{array}
$$

Remark 3.12. Under $C_{-}=0, C_{+}>0, G>0$ and $\eta_{+}>0$ in the model (3.5), we may recover a model driven by a gamma process of Kawai and Takeuchi [12] by formally taking $\alpha_{+} \downarrow 0$. However, the representation (2.10) for $\widetilde{L}_{+, T}$ does not hold for $\alpha_{+}=0$. Moreover, our Greeks formulas do not reduce to those obtained in [12], since we here rely on the exponential random variable $E_{+}$, which partially forms the stable random variable $\widetilde{L}_{+, T}$, irrelevant to the Girsanov transform with gamma processes adopted in [12]. 


\section{Numerical Illustrations}

We first test our Greeks formulas of the FMLS model (3.1) given in Theorem 3.1 on an out-of-the-money digital put payoff $\Phi(x)=\mathbb{1}(x \leq K)$. The model parameters are set $S_{0}=100, T=0.004, K=88, \alpha=1.5597, \sigma=0.1486, r=0.01$ and $q=0.005$. This setting ensures that the payoff function $\Phi(x)$ vanishes around $S_{0} e^{\left(r-q-c_{1}(\sigma)\right) T}$. In Figure1, we draw the Monte Carlo convergence of our formulas and the finite difference approximations with perturbation parameter $\varepsilon>0$ in, for example,

$$
\begin{gathered}
\frac{\partial}{\partial S_{0}} \mathbb{E}_{\mathbb{P}}\left[\Phi\left(S_{T}^{(\mathrm{s})}\right)\right] \approx \frac{1}{2 \varepsilon S_{0}}\left(\mathbb{E}_{\mathbb{P}}\left[\Phi\left((1+\varepsilon) S_{T}^{(\mathrm{s})}\right)\right]-\mathbb{E}_{\mathbb{P}}\left[\Phi\left((1-\varepsilon) S_{T}^{(\mathrm{ss})}\right)\right]\right), \\
\frac{\partial^{2}}{\partial S_{0}^{2}} \mathbb{E}_{\mathbb{P}}\left[\Phi\left(S_{T}^{(\mathrm{s})}\right)\right] \approx \frac{1}{\left(\varepsilon S_{0}\right)^{2}}\left(\mathbb{E}_{\mathbb{P}}\left[\Phi\left((1+\varepsilon) S_{T}^{(\mathrm{s})}\right)\right]-2 \mathbb{E}_{\mathbb{P}}\left[\Phi\left(S_{T}^{(\mathrm{s})}\right)\right]+\mathbb{E}_{\mathbb{P}}\left[\Phi\left((1-\varepsilon) S_{T}^{(\mathrm{s})}\right)\right]\right),
\end{gathered}
$$

and so on. The quantity $\frac{(\mathrm{FD})}{(\mathrm{MC})}$ indicates the variance ratio $\frac{\operatorname{Var}(\text { Finite Difference) }}{\operatorname{Var}(\text { Malliavin Calculus })}$. The figures and the variance ratios indicate a faster convergence of our formulas relative to the finite difference approximations. Recall that the finite difference approximations produce a biased estimate unless the payoff function is simply linear with respect to the parameter of interest.
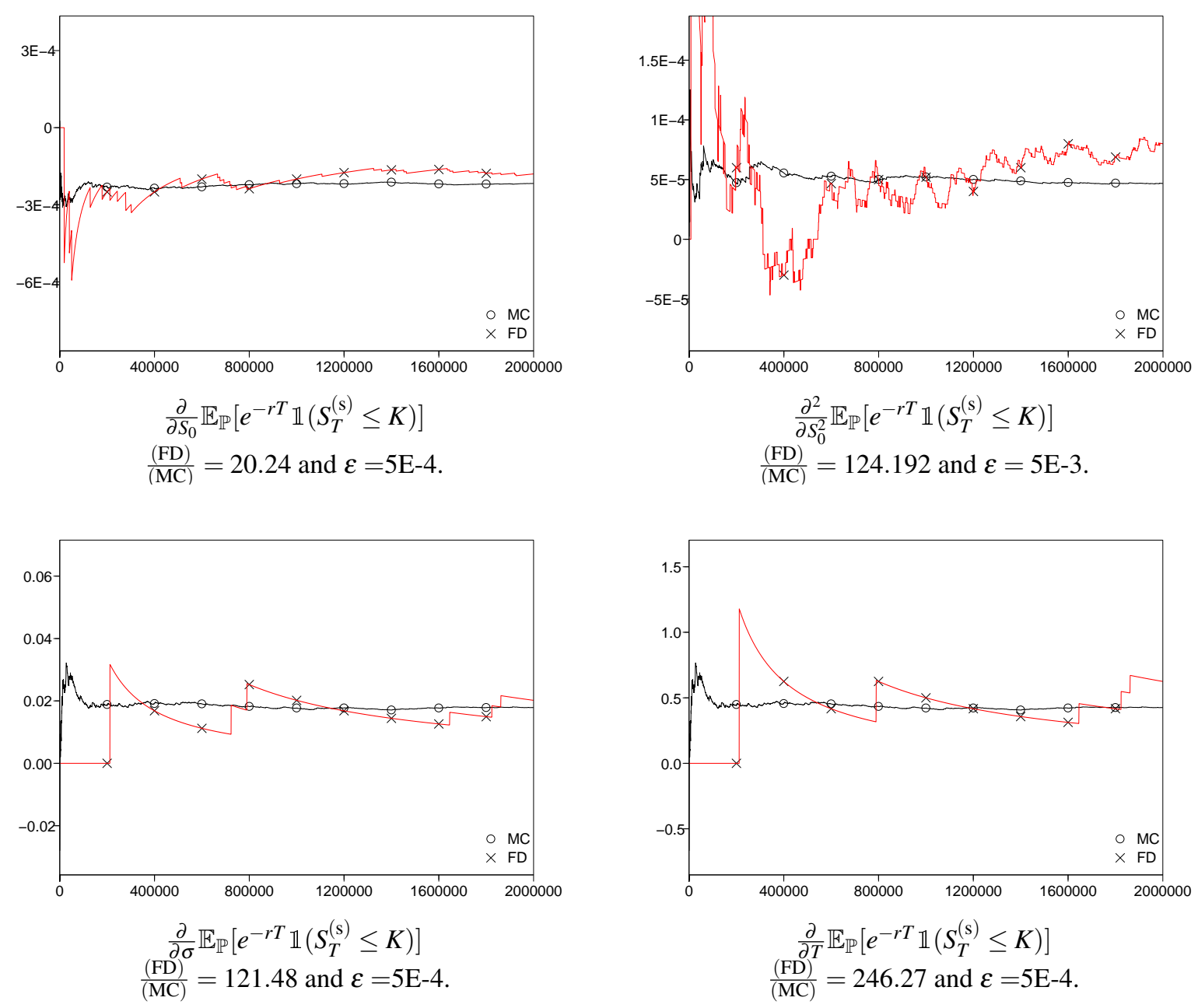

Figure 1: Numerical results for FMLS model. The model parameters are set $S_{0}=100, T=0.004, K=88, \alpha=1.5597$, $\sigma=0.1486, r=0.01$ and $q=0.005$.

Next, we test our Greeks formulas given in Theorem 3.7 of the tempered stable model (3.5) on an out-of-the-money digital put payoff $\Phi(x)=\mathbb{1}(x \leq K)$. The model parameters are set $S_{0}=100, T=0.004, K=80,\left(C_{-}, C_{+}, G, M, \alpha_{-}, \alpha_{+}\right)=$ 
$(0.5,0.5,1,4,0.5,1.2), \sigma=1, r=0.01$ and $q=0.005$. This setting ensures that the payoff function $\Phi(x)$ vanishes around $S_{0} e^{\left(r-q+\gamma_{2}-c_{2}(\sigma)\right) T}$. In Figure 2, we draw the Monte Carlo convergence of our formulas and the finite difference approximations as well.
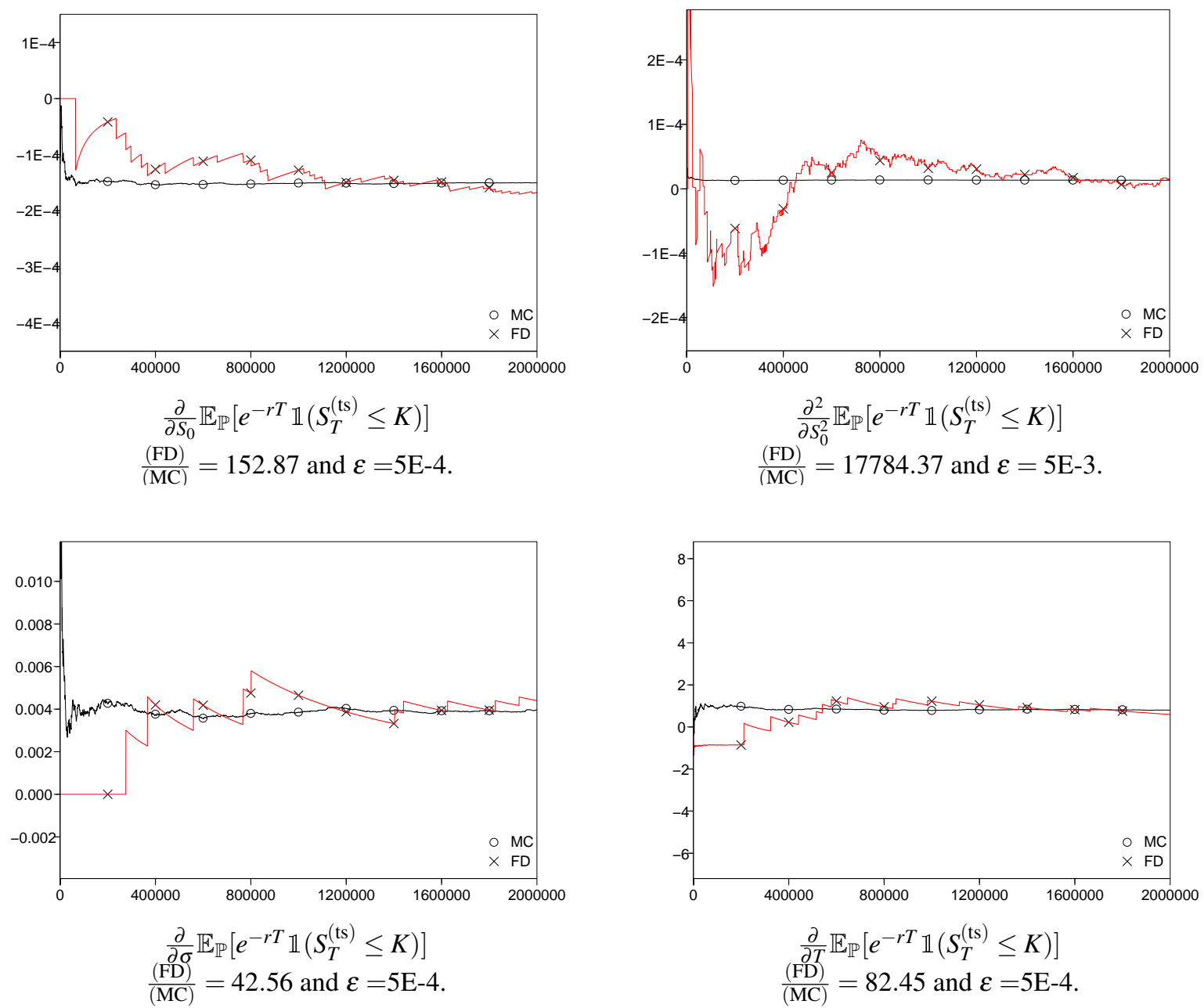

Figure 2: Numerical results for tempered stable process model. The model parameters are set $S_{0}=100, T=0.004$, $K=80,\left(C_{-}, C_{+}, G, M, \alpha_{-}, \alpha_{+}\right)=(0.5,0.5,1,4,0.5,1.2), \sigma=1, r=0.01$ and $q=0.005$. The parameters for the density transform are set $\eta_{-}=\eta_{+}=1$.

As discussed in Remark 3.9 the choice of $\left(\eta_{-}, \eta_{+}\right)$is arbitrary as long as $\eta_{-}, \eta_{+} \in[0,1]$ and $\eta_{-}+\eta_{+}>0$. We provide some numerical results in Table 1, 2 and 3 to illustrate how the choice affects the estimator variance, where the same model parameters are set as for the numerical experiments related to Figure 2 On one hand, in the put option setting in Table 1, the variance of our Delta and Gamma formulas can be reduced by setting $\eta_{+}$small, that is, by suppressing the integration-by-parts for the positive jump component $\widetilde{L}_{+, T}$. In the call option setting in Table 2, on the other hand, the variance is lower with a smaller $\eta_{-}$. Finally, in an artificial setting in Table 3, it seems reasonable to keep some effects from both components $\widetilde{L}_{-, T}$ and $\widetilde{L}_{+, T}$. Although generalizing based on numerical results is somewhat risky, it would be a natural conjecture that we should keep more effect from components to which the payoff is more sensitive. The choice of $\left(\eta_{-}, \eta_{+}\right)$should be different for different settings, while some pilot run would help. 


\begin{tabular}{c||c|c|c|c|c|c|c|c|c}
$\left(\eta_{-}, \eta_{+}\right)$ & $(0,1)$ & $(0.25,1)$ & $(0.5,1)$ & $(0.75,1)$ & $(1,1)$ & $(1,0.75)$ & $(1,0.5)$ & $(1,0.25)$ & $(1,0)$ \\
\hline \hline Delta & $1 \mathrm{E}-4$ & 28 & 85 & 128 & 153 & 171 & 187 & 196 & 198 \\
\hline Gamma & $7 \mathrm{E}-13$ & 550 & 5715 & 12679 & 17784 & 21959 & 25853 & 28632 & 29310
\end{tabular}

Table 1: Variance ratios for different choices of $\left(\eta_{-}, \eta_{+}\right)$on the digital put $\Phi(x)=\mathbb{1}(x \leq 80)$.

\begin{tabular}{c||c|c|c|c|c|c|c|c|c}
$\left(\eta_{-}, \eta_{+}\right)$ & $(0,1)$ & $(0.25,1)$ & $(0.5,1)$ & $(0.75,1)$ & $(1,1)$ & $(1,0.75)$ & $(1,0.5)$ & $(1,0.25)$ & $(1,0)$ \\
\hline \hline Delta & 15.69 & 15.14 & 13.54 & 11.49 & 9.47 & 7.21 & 4.30 & 1.36 & $1 \mathrm{E}-6$ \\
\hline Gamma & 31.74 & 31.46 & 28.47 & 22.82 & 16.28 & 9.33 & 3.00 & 0.25 & $1 \mathrm{E}-14$
\end{tabular}

Table 2: Variance ratios for different choices of $\left(\eta_{-}, \eta_{+}\right)$on the digital call $\Phi(x)=\mathbb{1}(x>120)$.

\begin{tabular}{c||c|c|c|c|c|c|c|c|c}
$\left(\eta_{-}, \eta_{+}\right)$ & $(0,1)$ & $(0.25,1)$ & $(0.5,1)$ & $(0.75,1)$ & $(1,1)$ & $(1,0.75)$ & $(1,0.5)$ & $(1,0.25)$ & $(1,0)$ \\
\hline \hline Delta & $3 \mathrm{E}-4$ & 18.40 & 20.00 & 17.56 & 14.72 & 11.35 & 6.84 & 2.18 & $1 \mathrm{E}-6$ \\
\hline Gamma & $2 \mathrm{E}-12$ & 48.76 & 45.51 & 41.06 & 26.08 & 14.95 & 4.79 & 0.41 & $1 \mathrm{E}-14$
\end{tabular}

Table 3: Variance ratios for different choices of $\left(\eta_{-}, \eta_{+}\right)$on an artificial payoff $\Phi(x)=\mathbb{1}\left(x \in \mathbb{R}_{+} \backslash(80,120]\right)$.

\section{Concluding Remarks}

In this paper, we have derived the Greeks formulas for derivative securities with both continuous and discontinuous European payoff structures under asset price dynamics described by stable and tempered stable processes of high practical interest. We have adopted the Malliavin calculus for jump processes by making use of a scaling property of exponential distributions with respect to the Girsanov density transform, which appear in the representation of stable distributions based on uniform and exponential distributions. Our approach allows the asset price process to be of a pure-jump type and incorporate infinite jump activities. The presentation of our Greeks formulas provides a direct help for practical use in particular of the tempered stable process setting, whose sample generation is in general not straightforward. We have also provided numerical results to illustrate that our Greeks formulas have faster rate of convergence relative to the finite difference approximations.

As future research, it would be interesting to investigate the application of the proposed approach to payoff functions with path dependent structure and the multi-asset cases. From both theoretical and practical points of view, it would be worthwhile to investigate dynamic hedging errors when using our Greeks formulas. Moreover, it would also be interesting to compare with finite difference approximations with a regularization of discoutinuous payoff functions. Those topics will be investigated in subsequence papers.

\section{A Proofs}

Let us begin with some notations and their basic properties, which will be used throughout the proofs. Let $T>0$, let $\lambda, \lambda_{-}, \lambda_{+} \in$ $(-1,+1)$ and let $\eta_{-}, \eta_{+} \in[0,1]$ such that $\eta_{-}+\eta_{+}>0$. Define

$$
\begin{gathered}
E_{-}^{(\lambda)}:=\frac{E_{-}}{1-\lambda}, \quad \widetilde{L}_{-, T}^{(\lambda)}:=(1-\lambda)^{\frac{1-\alpha_{-}}{\alpha_{-}}} \widetilde{L}_{-, T}, \quad \widetilde{S}_{T}^{(\mathrm{s}, \lambda)}:=S_{0} e^{\left(r-q-c_{1}(\sigma)\right) T+\sigma \widetilde{L}_{-, T}^{(\lambda)}}, \\
E_{+}^{(\lambda)}:=\frac{E_{+}}{1-\lambda}, \quad \widetilde{L}_{+, T}^{(\lambda)}:=(1-\lambda)^{\frac{1-\alpha_{+}}{\alpha_{+}}} \widetilde{L}_{+, T}, \quad \widetilde{S}_{T}^{\left(\mathrm{ts}, \lambda_{-}, \lambda_{+}\right)}:=S_{0} e^{\left(r-q+\gamma_{-}-c_{2}(\sigma)\right) T+\sigma \widetilde{L}_{-, T}^{(\lambda)}+\sigma \widetilde{L}_{+, T}^{\left(\lambda_{+}\right)}}
\end{gathered}
$$

and

$$
\begin{gathered}
J_{T}^{(\lambda)}:=\eta_{-}\left(1-E_{-}^{\left(\eta_{-} \lambda\right)}-\frac{1-\alpha_{-}}{\alpha_{-}} G \widetilde{L}_{-, T}^{\left(\eta_{-} \lambda\right)}\right)+\eta_{+}\left(1-E_{+}^{\left(\eta_{+} \lambda\right)}+\frac{1-\alpha_{+}}{\alpha_{+}} M \widetilde{L}_{+, T}^{\left(\eta_{+} \lambda\right)}\right), \\
K_{T}^{(\lambda)}:=\eta_{-} \frac{1-\alpha_{-}}{\alpha_{-}} \widetilde{L}_{-, T}^{\left(\eta_{-} \lambda\right)}+\eta_{+} \frac{1-\alpha_{+} \widetilde{L}_{+, T}^{\left(\eta_{+} \lambda\right)}}{\alpha_{+}} \\
N_{T}^{(\lambda)}:=\eta_{-}^{2}\left(\frac{1-\alpha_{-}}{\alpha_{-}}\right)^{2} \widetilde{L}_{-, T}^{\left(\eta_{-} \lambda\right)}+\eta_{+}^{2}\left(\frac{1-\alpha_{+}}{\alpha_{+}}\right)^{2} \widetilde{L}_{+, T}^{\left(\eta_{+} \lambda\right)} .
\end{gathered}
$$


Obviously, it holds almost surely that $E_{-}^{(0)}=E_{-}, E_{+}^{(0)}=E_{+}, \widetilde{L}_{-, T}^{(0)}=\widetilde{L}_{-, T}, \widetilde{L}_{+, T}^{(0)}=\widetilde{L}_{+, T}, \widetilde{S}_{T}^{(\mathrm{s}, 0)}=\widetilde{S}_{T}^{(\mathrm{s})}, \widetilde{S}_{T}^{(\mathrm{ts}, 0,0)}=\widetilde{S}_{T}^{(\mathrm{ts})}, J_{T}^{(0)}=J_{T}$, $K_{T}^{(0)}=K_{T}$ and $N_{T}^{(0)}=N_{T}$. Moreover, in a similar manner to 2.2 with $a=b=1$, define the Radon-Nikodým derivative

$$
\left.\frac{d \mathbb{Q}_{\lambda}}{d \mathbb{P}}\right|_{\mathscr{G}_{2}}:=\frac{e^{\lambda E_{-}}}{\mathbb{E}_{\mathbb{P}}\left[e^{\lambda E_{-}}\right]}=(1-\lambda) e^{\lambda E_{-}}, \quad \mathbb{P} \text {-a.s. }
$$

where $\mathscr{G}_{2}$ is the minimal $\sigma$-field generated by the random variable $E_{-}$. Also, define for $\eta_{-} \in[0,1]$ and $\eta_{+} \in[0,1]$,

$$
\left.\frac{d \mathbb{Q}_{\lambda}}{d \mathbb{Q}_{0}}\right|_{\mathscr{G}_{3}}:=\frac{e^{\eta_{-} \lambda E_{-}+\eta_{+} \lambda E_{+}}}{\mathbb{E}_{\mathbb{P}}\left[e^{\eta_{-} \lambda E_{-}+\eta_{+} \lambda E_{+}}\right]}=\left(1-\eta_{-} \lambda\right)\left(1-\eta_{+} \lambda\right) e^{\eta_{-} \lambda E_{-}+\eta_{+} \lambda E_{+}}, \quad \mathbb{Q}_{0} \text {-a.s. }
$$

where $\mathscr{G}_{3}$ is the minimal $\sigma$-field generated by the random variables $E_{-}$and $E_{+}$.

Lemma A.1. (i) We have

$$
\begin{gathered}
\frac{\partial}{\partial \lambda} E_{-}^{(\lambda)}=\frac{E_{-}^{(\lambda)}}{1-\lambda}, \quad \frac{\partial}{\partial \lambda} \widetilde{L}_{-, T}^{(\lambda)}=-\frac{1-\alpha_{-}}{\alpha_{-}} \frac{\widetilde{L}_{-, T}^{(\lambda)}}{1-\lambda}, \quad \frac{\partial}{\partial \lambda} \widetilde{S}_{T}^{(\mathrm{s}, \lambda)}=-\sigma \frac{1-\alpha_{-}}{\alpha_{-}} \frac{\widetilde{L}_{-, T}^{(\lambda)}}{1-\lambda} \widetilde{S}_{t}^{(\mathrm{s}, \lambda)}, \\
\frac{\partial}{\partial \lambda} E_{+}^{(\lambda)}=\frac{E_{+}^{(\lambda)}}{1-\lambda}, \quad \frac{\partial}{\partial \lambda} \widetilde{L}_{+, T}^{(\lambda)}=-\frac{1-\alpha_{+}}{\alpha_{+}} \frac{\widetilde{L}_{+, T}^{(\lambda)}}{1-\lambda},\left.\quad \frac{\partial}{\partial \lambda}\left(K_{T}^{(\lambda)}\right)\right|_{\lambda=0}=-N_{T},
\end{gathered}
$$

and

$$
\frac{\partial}{\partial \lambda} \widetilde{S}_{T}^{\left(\mathrm{ts}, \eta_{-} \lambda, \eta_{+} \lambda\right)}=-\sigma\left(\frac{1-\alpha_{-}}{\alpha_{-}} \frac{\eta_{-}}{1-\eta_{-} \lambda} \widetilde{L}_{-, T}^{\left(\eta_{-} \lambda\right)}+\frac{1-\alpha_{+}}{\alpha_{+}} \frac{\eta_{+}}{1-\eta_{+} \lambda} \widetilde{L}_{+, T}^{\left(\eta_{+} \lambda\right)}\right) \widetilde{S}_{T}^{\left(\mathrm{ts}, \eta_{-} \lambda, \eta_{+} \lambda\right)},
$$

where each equality holds under its suitable probability measure. Moreover,

$$
\begin{gathered}
\frac{\partial}{\partial \lambda}\left(\left.\frac{d \mathbb{Q}_{\lambda}}{d \mathbb{P}}\right|_{\mathscr{G}_{2}}\right)=\left(E_{-}-\frac{1}{1-\lambda}\right)\left(\left.\frac{d \mathbb{Q}_{\lambda}}{d \mathbb{P}}\right|_{\mathscr{G}_{2}}\right), \quad \mathbb{P} \text {-a.s. } \\
\frac{\partial}{\partial \lambda}\left(\left.\frac{d \mathbb{Q}_{\lambda}}{d \mathbb{Q}_{0}}\right|_{\mathscr{G}_{3}}\right)=\left[\eta_{-}\left(E_{-}-\frac{1}{1-\eta_{-} \lambda}\right)+\eta_{+}\left(E_{+}-\frac{1}{1-\eta_{+} \lambda}\right)\right]\left(\left.\frac{d \mathbb{Q}_{\lambda}}{d \mathbb{Q}_{0}}\right|_{\mathscr{G}_{3}}\right), \quad \mathbb{Q}_{0} \text {-a.s. },
\end{gathered}
$$

where the Radon-Nikodým derivatives are defined in A.1 and A.2.

(ii) For $q_{1} \in\left(1, \alpha_{-}\right)$and $q_{2} \in\left(1, \alpha_{+}\right)$,

$$
\mathbb{E}_{\mathbb{P}}\left[\left|L_{-, T}\right|^{q_{1}} e^{q_{1} \sigma L_{-, T}}\right]<+\infty, \quad \mathbb{E}_{\mathbb{P}}\left[\left|L_{+, T}\right|^{q_{2}} e^{-q_{2} \sigma L_{+, T}}\right]<+\infty .
$$

(iii) For $q_{3}>1$ and $q_{4}>1$,

$$
\mathbb{E}_{\mathbb{P}}\left[e^{q_{3} \sigma L_{-, T}}\right]<+\infty, \quad \mathbb{E}_{\mathbb{P}}\left[e^{-q_{4} \sigma L_{+, T}}\right]<+\infty .
$$

(iv) Let $\beta \in(0,1)$ and let $E$ be a standard exponential random variable under $\mathbb{P}$. It holds that for $q \in(1,1 / \beta)$,

$$
\mathbb{E}_{\mathbb{P}}\left[E^{q}\right]<+\infty, \quad \mathbb{E}_{\mathbb{P}}\left[e^{q \beta E}\right]<+\infty, \quad \mathbb{E}_{\mathbb{P}}\left[E^{q} e^{q \beta E}\right]<+\infty .
$$

(v) The marginals $L_{-, T}, L_{+, T}, X_{-, T}^{(\mathrm{ts})}$ and $X_{+, T}^{(\mathrm{ts})}$ have $C^{\infty}$-densities.

Proof. (i) The differentiations with respect to the parameter $\lambda$ are justified since they are all independent of every random element. The simple computation yields the results.

(ii) It suffices to prove for $L_{-, T}$. First, we have

$$
\begin{aligned}
\mathbb{E}_{\mathbb{P}}\left[\left|L_{-, T}\right|^{q_{1}} e^{q_{1} \sigma L_{-, T}} ; L_{-, T} \in \mathbb{R}_{+}\right] & =\mathbb{E}_{\mathbb{P}}\left[\int_{0}^{L_{-, T}}\left(x^{q_{1}} e^{q_{1} \sigma x}\right)^{\prime} d x ; L_{-, T} \in \mathbb{R}_{+}\right] \\
& =\mathbb{E}_{\mathbb{P}}\left[\int_{0}^{+\infty} q_{1}\left(x^{q_{1}-1}+\sigma x^{q_{1}}\right) e^{q_{1} \sigma x_{1}} \mathbb{1}_{[x,+\infty)}\left(L_{-, T}\right) d x\right] \\
& =\int_{0}^{+\infty} q_{1}\left(x^{q_{1}-1}+\sigma x^{q_{1}}\right) e^{q_{1} \sigma x} \mathbb{P}\left(L_{-, T}>x\right) d x<+\infty,
\end{aligned}
$$

where we have used the Fubini theorem due to the non-negative integrand, and where the inequality holds by the tail behavior 2.8 and by $\alpha_{-} /\left(1-\alpha_{-}\right) \in(2,+\infty)$. Next,

$$
\begin{aligned}
\mathbb{E}_{\mathbb{P}}\left[\left|L_{-, T}\right|^{q_{1}} e^{q_{1} \sigma L_{-, T}} ; L_{-, T} \in \mathbb{R}_{-}\right] & \leq \mathbb{E}_{\mathbb{P}}\left[\left(-L_{-, T}\right)^{q_{1}} ; L_{-, T} \in \mathbb{R}_{-}\right] \\
& =\mathbb{E}_{\mathbb{P}}\left[\int_{0}^{+\infty} q_{1} x^{q_{1}-1} \mathbb{1}_{[x,+\infty)}\left(-L_{-, T}\right) d x\right] \\
& =\int_{0}^{+\infty} q_{1} x^{q_{1}-1} \mathbb{P}\left(L_{-, T} \leq-x\right) d x<+\infty,
\end{aligned}
$$


where we have again used the Fubini theorem due to the non-negative integrand, and where the inequality holds by the tail behavior (2.7).

(iii) The proof can be completed in a similar manner to that of (ii).

(iv) A direct computation shows the result with the exponential density function.

(v) The marginals $L_{-, T}$ and $L_{+, T}$ have stable distributions, while $X_{-, T}^{(\mathrm{ts})}$ and $X_{+, T}^{(\mathrm{ts})}$ have tempered stable distributions. Thus, they are in the class of selfdecomposable distributions. The claim holds by

$$
\lim _{z \uparrow 0}|z| \frac{v_{(\mathrm{s},-)}(d z)}{d z}=+\infty, \quad \lim _{z \downarrow 0}|z| \frac{v_{(\mathrm{s},+)}(d z)}{d z}=+\infty,
$$

and

$$
\lim _{z \uparrow 0}|z| \frac{v_{(\mathrm{ts},-)}(d z)}{d z}=+\infty, \quad \lim _{z \downarrow 0}|z| \frac{v_{(\mathrm{ts},+)}(d z)}{d z}=+\infty,
$$

where the Lévy measures are defined as (2.6) and (3.4), with the help of Theorem 28.4 of Sato [18].

To prove Theorem 3.1 the following is useful and very insightful.

Proposition A.2. (i) It holds that

$$
\frac{\sigma\left(1-\alpha_{-}\right)}{\alpha_{-}} \mathbb{E}_{\mathbb{P}}\left[\Phi^{\prime}\left(S_{T}^{(\mathrm{s})}\right) S_{T}^{(\mathrm{s})} L_{-, T}\right]=\mathbb{E}_{\mathbb{P}}\left[\Phi\left(\widetilde{S}_{T}^{(\mathrm{s})}\right)\left(1-E_{-}\right)\right]
$$

(ii) It holds that for each $x>0$ and $\varepsilon>0$ such that $x>\varepsilon$ and for each compact set $H \subset \mathbb{R}_{+}^{3}\left(:=\mathbb{R}_{+} \times \mathbb{R}_{+} \times \mathbb{R}_{+}\right)$,

$$
\sup _{\left(S_{0}, \sigma, T\right) \in H} \mathbb{P}\left(S_{T}^{(\mathrm{s})} \in[x-\varepsilon, x+\varepsilon]\right) \leq C_{H} \ln \frac{x+\varepsilon}{x-\varepsilon},
$$

where $C_{H}$ is a positive constant depending on $H$.

Proof. (i) By the scaling property (2.3), it holds that

$$
\mathbb{E}_{\mathbb{P}}\left[\Phi\left(\widetilde{S}_{T}^{(\mathrm{s}, \lambda)}\right)\right]=\mathbb{E}_{\mathbb{P}}\left[\left.\frac{d \mathbb{Q}_{\lambda}}{d \mathbb{P}}\right|_{\mathscr{G}_{2}} \Phi\left(\widetilde{S}_{T}^{(\mathrm{s})}\right)\right],
$$

where the Radon-Nikodým derivative is defined by A.1. Then, by differentiating the both hand sides with respect to $\lambda$ at the origin, we get the result. The interchange of the derivative and the integral are justified as follows.

We begin with the left hand side of $\mathrm{A} .3$. Let $\lambda \in[0,1 / 2]$ and $\varepsilon \in(-\lambda,+\lambda)$. By the Taylor theorem, it holds that

$$
\frac{\Phi\left(\widetilde{S}_{T}^{(\mathrm{s}, \varepsilon)}\right)-\Phi\left(\widetilde{S}_{T}^{(\mathrm{s})}\right)}{\varepsilon}=\frac{\sigma\left(\alpha_{-}-1\right)}{\alpha_{-}} \int_{0}^{1} \frac{\Phi^{\prime}\left(\widetilde{S}_{T}^{(\mathrm{s}, \delta \varepsilon)}\right) \widetilde{S}_{T}^{(\mathrm{s}, \delta \varepsilon)} \widetilde{L}_{-, T}^{(\delta \varepsilon)}}{1-\delta \varepsilon} d \delta, \quad \mathbb{P} \text {-a.s. }
$$

Hence, for conjugate exponents $\left(p_{1}, p_{1}^{\prime}\right)$ and $\left(p_{2}, p_{2}^{\prime}\right)$ with $p_{1} \in\left(1, \alpha_{-}\right)$and $p_{2} \in\left(1, \alpha_{-} / \sqrt{2}\right)$, the Hölder inequality yields

$$
\begin{aligned}
& \mathbb{E}_{\mathbb{P}} {\left[\left|\frac{\Phi\left(\widetilde{S}_{T}^{(\mathrm{s}, \varepsilon)}\right)-\Phi\left(\widetilde{S}_{T}^{(\mathrm{s})}\right)}{\varepsilon}-\frac{\sigma\left(\alpha_{-}-1\right)}{\alpha_{-}} \Phi^{\prime}\left(\widetilde{S}_{T}^{(\mathrm{s})}\right) \widetilde{S}_{T}^{(\mathrm{s})} \widetilde{L}_{-, T}\right|\right] } \\
&= \frac{\sigma\left(\alpha_{-}-1\right)}{\alpha_{-}} \mathbb{E}_{\mathbb{P}}\left[\left|\int_{0}^{1} \frac{\Phi^{\prime}\left(\widetilde{S}_{T}^{(\mathrm{s}, \delta \varepsilon)}\right) \widetilde{S}_{T}^{(\mathrm{s}, \delta \varepsilon)} \widetilde{L}_{-, T}^{(\delta \varepsilon)}}{1-\delta \varepsilon} d \delta-\Phi^{\prime}\left(\widetilde{S}_{T}^{(\mathrm{s})}\right) \widetilde{S}_{T}^{(\mathrm{s})} \widetilde{L}_{-, T}\right|\right] \\
& \leq \frac{\sigma\left(\alpha_{-}-1\right)}{\alpha_{-}}\left(\int_{0}^{1} \mathbb{E}_{\mathbb{P}}\left[\left|\Phi^{\prime}\left(\widetilde{S}_{T}^{(\mathrm{s}, \delta \varepsilon)}\right)-\Phi^{\prime}\left(\widetilde{S}_{T}^{(\mathrm{s})}\right)\right|^{p_{1}^{\prime}}\right]^{1 / p_{1}^{\prime}} \mathbb{E}_{\mathbb{P}}\left[\left|\widetilde{S}_{T}^{(\mathrm{s})} \widetilde{L}_{-, T}\right|^{p_{1}}\right]^{1 / p_{1}} d \delta\right. \\
&\left.\left.+\int_{0}^{1} \mathbb{E}_{\mathbb{P}}\left[\left|\Phi^{\prime}\left(\widetilde{S}_{T}^{(\mathrm{s}, \delta \varepsilon)}\right)\right|^{p_{2}^{\prime}}\right]^{1 / p_{2}^{\prime}} \mathbb{E}_{\mathbb{P}}\left[\mid \frac{\widetilde{S}_{T}^{(\mathrm{s}, \delta \varepsilon)} \widetilde{L}_{-, T}^{(\delta \varepsilon)}}{1-\delta \varepsilon}-\widetilde{S}_{T}^{(\mathrm{s})} \widetilde{L}_{-, T}^{p_{2}}\right]^{1 / p_{2}}\right] \delta\right)
\end{aligned}
$$

It suffices to show that the above terms A.4 and A.5 tend to zero as $\varepsilon \downarrow 0$. For the first term of A.4, the limit and the expectation can be interchanged due to the boundedness of $\Phi^{\prime}$. Moreover, since $\Phi^{\prime}$ is continuous and $\lim _{\mathcal{\varepsilon} \downarrow 0} \widetilde{S}_{T}^{(\mathrm{s}, \varepsilon)}=\widetilde{S}_{T}^{(\mathrm{s})}, \mathbb{P}$-a.s., the first term tends to zero as $\varepsilon \downarrow 0$. Moreover, it holds by Lemma A.1 (ii) that

$$
\mathbb{E}_{\mathbb{P}}\left[\left|\widetilde{S}_{T}^{(\mathrm{s})} \widetilde{L}_{-, T}\right|^{p_{1}}\right]=\left(S_{0} e^{\left(r-q-c_{1}(\sigma)\right) T}\right)^{p_{1}} \mathbb{E}_{\mathbb{P}}\left[\left|L_{-, T}\right|^{p_{1}} e^{p_{1} \sigma L_{-, T}}\right]<+\infty .
$$


Therefore, the term A.4 tends to zero as $\varepsilon \downarrow 0$. Next, the first term of A.5 is finite again due to the boundedness of $\Phi^{\prime}$. For the second term of (A.5), it holds by Lemma A.1 (ii) that for every $\varepsilon \in(-\lambda,+\lambda)$ and $\gamma \in(1, \sqrt{2})$,

$$
\mathbb{E}_{\mathbb{P}}\left[\left|\frac{\widetilde{S}_{T}^{(\mathrm{s}, \delta \varepsilon)} \widetilde{L}_{-, T}^{(\delta \varepsilon)}}{1-\delta \varepsilon}\right|^{p_{2} \gamma}\right] \leq\left(2^{\frac{2 \alpha_{-}-1}{\alpha_{-}}} S_{0} e^{\left(r-q-c_{1}(\sigma)\right) T}\right)^{p_{2} \gamma} \mathbb{E}_{\mathbb{P}}\left[\left|L_{-, T}\right|^{p_{2}} e^{p_{2} \gamma \sigma(1-\delta \varepsilon)^{\frac{1-\alpha_{-}}{\alpha_{-}}} L_{-, T}}\right]<+\infty
$$

since $(1-\delta \varepsilon)^{\left(1-\alpha_{-}\right) / \alpha_{-}}<\sqrt{2}$. Hence, the limit and the expectation can be interchanged, and thus the term A.5 also tends to zero as $\varepsilon \downarrow 0$.

Next, for the right hand side of $\mathrm{A.3}$, observe first that

$$
\frac{(1-\varepsilon) e^{\varepsilon E_{-}}-1}{\varepsilon}=\int_{0}^{1}\left((1-\delta \varepsilon) E_{-}-1\right) e^{\delta \varepsilon E_{-}} d \delta, \quad \mathbb{P}-\text { a.s. }
$$

For conjugate exponents $(p, q)$ with $q \in(1,1 /(\delta \varepsilon))$, the Hölder inequality yields

$$
\begin{aligned}
\mathbb{E}_{\mathbb{P}}\left[\left|\Phi\left(\widetilde{S}_{T}^{(\mathrm{s})}\right) \frac{(1-\varepsilon) e^{\varepsilon E_{-}}-1}{\varepsilon}-\Phi\left(\widetilde{S}_{T}^{(\mathrm{s})}\right)\left(E_{-}-1\right)\right|\right] \\
\quad \leq \mathbb{E}_{\mathbb{P}}\left[\left|\Phi\left(\widetilde{S}_{T}^{(\mathrm{s})}\right)\right|^{p}\right]^{1 / p} \int_{0}^{1} \mathbb{E}_{\mathbb{P}}\left[\left|\left((1-\delta \varepsilon) E_{-}-1\right) e^{\delta \varepsilon E_{-}}-\left(E_{-}-1\right)\right|^{q}\right]^{1 / q} d \delta .
\end{aligned}
$$

For the first term, there exists a constant $C_{p}>0$ depending on $p$ such that

$$
\begin{aligned}
\mathbb{E}_{\mathbb{P}}\left[\left|\Phi\left(\widetilde{S}_{T}^{(\mathrm{s})}\right)\right|^{p}\right] & \leq C_{p} \mathbb{E}_{\mathbb{P}}\left[\left|\Phi\left(S_{T}^{(\mathrm{s})}\right)-\Phi\left(S_{0}\right)\right|^{p}\right]+C_{p}\left|\Phi\left(S_{0}\right)\right|^{p} \\
& \leq C_{p}\left\|\Phi^{\prime}\right\|_{\infty}^{p} \mathbb{E}_{\mathbb{P}}\left[\left|S_{T}^{(\mathrm{s})}-S_{0}\right|^{p}\right]+C_{p}\left|\Phi\left(S_{0}\right)\right|^{p}<+\infty,
\end{aligned}
$$

where the second inequality holds by the mean value theorem. We can prove that the second term tends to zero as $\varepsilon \downarrow 0$ in a similar manner, with the help of LemmaA.1(ii). This completes the proof of (i).

(ii) By Lemma A.1(v), the marginal $L_{-, T}$ has the $C^{\infty}$-density. By denoting by $f$ the density, we have

$$
\mathbb{P}\left(S_{T}^{(\mathrm{s})} \in[x-\varepsilon, x+\varepsilon]\right)=\mathbb{P}\left(L_{-, T} \in\left[b_{-}(x), b_{+}(x)\right]\right)=\int_{b_{-}(x)}^{b_{+}(x)} f(y) d y \leq\left(\frac{1}{\sigma_{y \in\left[b_{-}(x), b_{+}(x)\right]}} \sup _{f(y))} \ln \frac{x+\varepsilon}{x-\varepsilon},\right.
$$

where $b_{ \pm}(x):=\sigma^{-1}\left(\ln \left((x \pm \varepsilon) / S_{0}\right)-\left(r-q-c_{1}(\sigma)\right) T\right)$. This completes the proof.

Proof of Theorem 3.1 First, assume that the function $\Phi$ is in $C_{b}^{1}\left(\mathbb{R}_{+} ; \mathbb{R}\right)$ and vanishes on a neighborhood of $x_{0}:=S_{0} e^{\left(r-q-c_{1}(\sigma)\right) T}$. For the Delta formula, instead of the identity A.3, we are only to differentiate the both sides of

$$
\mathbb{E}_{\mathbb{P}}\left[\frac{\Phi\left(\widetilde{S}_{T}^{(\mathrm{s}, \lambda)}\right)}{\widetilde{L}_{-, T}^{(\lambda)}}\right]=\mathbb{E}_{\mathbb{P}}\left[\left.\frac{d \mathbb{Q}_{\lambda}}{d \mathbb{P}}\right|_{\mathscr{G}_{2}} \frac{\Phi\left(\widetilde{S}_{T}^{(\mathrm{s})}\right)}{\widetilde{L}_{-, T}}\right]
$$

with respect to $\lambda$ at the origin, where the Radon-Nikodým derivative is defined by A.1. The interchange of the derivative and the expectation can be justified by the dominated convergence theorem, just in a similar manner to the proof of Proposition A.2 (i), since the random variable $\Phi\left(\widetilde{S}_{T}^{(\mathrm{s}, \lambda)}\right)$ vanishes when both $\widetilde{L}_{-, T}^{(\lambda)}$ and $\widetilde{L}_{+, T}^{(\lambda)}$ are very close to the origin. For the Gamma formula, by making use of the Delta formula, we get

$$
\begin{aligned}
\frac{\partial^{2}}{\partial S_{0}^{2}} \mathbb{E}_{\mathbb{P}}\left[\Phi\left(S_{T}^{(\mathrm{s})}\right)\right] & =\frac{\partial}{\partial S_{0}}\left(\frac{\partial}{\partial S_{0}} \mathbb{E}_{\mathbb{P}}\left[\Phi\left(S_{T}^{(\mathrm{s})}\right)\right]\right) \\
& =\frac{\partial}{\partial S_{0}}\left(\frac{1}{S_{0}} \mathbb{E}_{\mathbb{P}}\left[\Phi\left(\widetilde{S}_{T}^{(\mathrm{s})}\right) \frac{1-\alpha_{-} E_{-}}{\sigma\left(1-\alpha_{-}\right) \widetilde{L}_{-, T}}\right]\right) \\
& =\frac{1}{S_{0}^{2}} \mathbb{E}_{\mathbb{P}}\left[\Phi^{\prime}\left(\widetilde{S}_{T}^{(\mathrm{s})}\right) \widetilde{S}_{T}^{(\mathrm{s})} \frac{1-\alpha_{-} E_{-}}{\sigma\left(1-\alpha_{-}\right) \widetilde{L}_{-, T}}\right]-\frac{1}{S_{0}} \frac{\partial}{\partial S_{0}} \mathbb{E}_{\mathbb{P}}\left[\Phi\left(S_{T}^{(\mathrm{s})}\right)\right] .
\end{aligned}
$$

The first term can be proceeded by differentiating the both sides of

$$
\mathbb{E}_{\mathbb{P}}\left[\Phi\left(\widetilde{S}_{T}^{(\mathrm{s}, \lambda)}\right) \frac{1-\alpha_{-} E_{-}^{(\lambda)}}{\left(\widetilde{L}_{-, T}^{(\lambda)}\right)^{2}}\right]=\mathbb{E}_{\mathbb{P}}\left[\left.\frac{d \mathbb{Q}_{\lambda}}{d \mathbb{P}}\right|_{\mathscr{G}_{2}} \Phi\left(\widetilde{S}_{T}^{(\mathrm{s})}\right) \frac{1-\alpha_{-} E_{-}}{\left(\widetilde{L}_{-, T}\right)^{2}}\right],
$$


with respect to $\lambda$ at the origin, where the Radon-Nikodým derivative is defined by A.1). For the Vega and Theta formulas,

$$
\begin{aligned}
& \frac{\partial}{\partial \sigma} \mathbb{E}_{\mathbb{P}}\left[\Phi\left(S_{T}^{(\mathrm{s})}\right)\right]=\mathbb{E}_{\mathbb{P}}\left[\Phi^{\prime}\left(S_{T}^{(\mathrm{s})}\right) S_{T}^{(\mathrm{s})} L_{-, T}\right]-T \frac{\alpha_{-} c_{1}(\sigma)}{\sigma} \mathbb{E}_{\mathbb{P}}\left[\Phi^{\prime}\left(S_{T}^{(\mathrm{s})}\right) S_{T}^{(\mathrm{s})}\right], \\
& \frac{\partial}{\partial T} \mathbb{E}_{\mathbb{P}}\left[\Phi\left(S_{T}^{(\mathrm{s})}\right)\right]=\frac{\sigma}{\alpha_{-} T} \mathbb{E}_{\mathbb{P}}\left[\Phi^{\prime}\left(S_{T}^{(\mathrm{s})}\right) S_{T}^{(\mathrm{s})} L_{-, T}\right]+\left(r-q-c_{1}(\sigma)\right) \mathbb{E}_{\mathbb{P}}\left[\Phi^{\prime}\left(S_{T}^{(\mathrm{s})}\right) S_{T}^{(\mathrm{s})}\right],
\end{aligned}
$$

where the first terms can again be proceeded with the help of Proposition A.2(i).

Next, we remove the regularity of the payoff function $\Phi$ and extend to the class $\mathscr{J}\left(\mathbb{R}_{+} ; \mathbb{R}\right)$. We only prove the case of the Delta formula. To approximate the function $\Phi \in \mathscr{E}_{1}$ by a sequence $\left\{\Phi_{k}\right\}_{k \in \mathbb{N}}$ of functions in $\mathscr{E}_{2}$ such that $\mathscr{E}_{2} \subset \mathscr{E}_{1}$, it suffices to show that for each compact set $H \subset \mathbb{R}_{+}^{3}$,

$$
\sup _{\left(S_{0}, \sigma, T\right) \in H}\left|\mathbb{E}_{\mathbb{P}}\left[\Phi_{k}\left(S_{T}^{(\mathrm{s})}\right)\right]-\mathbb{E}_{\mathbb{P}}\left[\Phi\left(S_{T}^{(\mathrm{s})}\right)\right]\right| \rightarrow 0
$$

and

$$
\sup _{\left(S_{0}, \sigma, T\right) \in H}\left|\frac{\partial}{\partial S_{0}} \mathbb{E}_{\mathbb{P}}\left[\Phi_{k}\left(S_{T}^{(\mathrm{s})}\right)\right]-\frac{1}{S_{0}} \mathbb{E}_{\mathbb{P}}\left[\Phi\left(\widetilde{S}_{T}^{(\mathrm{s})}\right) \frac{1-\alpha_{-} E_{-}}{\sigma\left(1-\alpha_{-}\right) \widetilde{L}_{-, T}}\right]\right| \rightarrow 0,
$$

as $k \uparrow+\infty$. To this end, suppose that the Delta formula holds for a sequence $\left\{\Phi_{k}\right\}_{k \in \mathbb{N}}$ of functions in $\mathscr{E}_{2}$. For conjugate exponent $\left(p_{1}, p_{2}\right)$ satisfying $p_{2} \in\left(1, \alpha_{-}\right)$and for each $\left(S_{0}, \sigma, T\right) \in H$, it holds by the Hölder inequality that

$$
\left|\mathbb{E}_{\mathbb{P}}\left[\Phi_{k}\left(S_{T}^{(\mathrm{s})}\right)\right]-\mathbb{E}_{\mathbb{P}}\left[\Phi\left(S_{T}^{(\mathrm{s})}\right)\right]\right| \leq \mathbb{E}_{\mathbb{P}}\left[\left|\frac{\Phi_{k}\left(S_{T}^{(\mathrm{s})}\right)-\Phi\left(S_{T}^{(\mathrm{s})}\right)}{S_{0} \sigma L_{-, T}}\right|^{p_{1}}\right]^{1 / p_{1}} \mathbb{E}_{\mathbb{P}}\left[\left|S_{0} \sigma L_{-, T}\right|^{p_{2}}\right]^{1 / p_{2}},
$$

and

$$
\begin{aligned}
\mid \frac{\partial}{\partial S_{0}} & \mathbb{E}_{\mathbb{P}}\left[\Phi_{k}\left(S_{T}^{(\mathrm{s})}\right)\right]-\mathbb{E}_{\mathbb{P}}\left[\Phi\left(\widetilde{S}_{T}^{(\mathrm{s})}\right) \frac{1-\alpha_{-} E_{-}}{S_{0} \sigma\left(1-\alpha_{-}\right) \widetilde{L}_{-, T}}\right] \mid \\
& =\left|\mathbb{E}_{\mathbb{P}}\left[\left(\Phi_{k}\left(\widetilde{S}_{T}^{(\mathrm{s})}\right)-\Phi\left(\widetilde{S}_{T}^{(\mathrm{s})}\right)\right) \frac{1-\alpha_{-} E_{-}}{S_{0} \sigma\left(1-\alpha_{-}\right) \widetilde{L}_{-, T}}\right]\right| \\
& \leq \mathbb{E}_{\mathbb{P}}\left[\left|\frac{\Phi_{k}\left(S_{T}^{(\mathrm{s})}\right)-\Phi\left(S_{T}^{(\mathrm{s})}\right)}{S_{0} \sigma L_{-, T}}\right|^{p_{1}}\right]^{1 / p_{1}} \mathbb{E}_{\mathbb{P}}\left[\left|\frac{1-\alpha_{-} E_{-}}{1-\alpha_{-}}\right|^{p_{2}}\right]^{1 / p_{2}} .
\end{aligned}
$$

Clearly, $\mathbb{E}_{\mathbb{P}}\left[\left|L_{-, T}\right|^{p_{2}}\right]<+\infty$ and $\mathbb{E}_{\mathbb{P}}\left[\left|1-\alpha_{-} E_{-}\right|^{p_{2}}\right]<+\infty$. Therefore, we are only to show that for $\Phi \in \mathscr{E}_{1}$ and a sequence $\left\{\Phi_{k}\right\}_{k \in \mathbb{N}}$ of functions in $\mathscr{E}_{2}$ such that $\mathscr{E}_{2} \subset \mathscr{E}_{1}$,

$$
\sup _{\left(S_{0}, \sigma, T\right) \in H} \mathbb{E}_{\mathbb{P}}\left[\left|\frac{\Phi_{k}\left(S_{T}^{(\mathrm{s})}\right)-\Phi\left(S_{T}^{(\mathrm{s})}\right)}{L_{-, T}}\right|^{p_{1}}\right] \rightarrow 0,
$$

as $k \uparrow+\infty$. Throughout the proof, $C_{H}$ is a positive constant depending on a set $H \subset \mathbb{R}_{+}^{3}$, which may change its value from line to line.

Step 1: First, we extend from $C_{b}^{1}\left(\mathbb{R}_{+} ; \mathbb{R}\right)$ to $C_{K}\left(\mathbb{R}_{+} ; \mathbb{R}\right)$. Clearly, $\Phi \in C_{K}\left(\mathbb{R}_{+} ; \mathbb{R}\right)$ vanishing around $x_{0}$ can be approximated uniformly and boundedly by a sequence $\left\{\Phi_{k}\right\}_{k \in \mathbb{N}}$ of functions in $C_{K}^{\infty}\left(\mathbb{R}_{+} ; \mathbb{R}\right)$ vanishing around $x_{0}$. Then, it holds that for each compact set $H \subset \mathbb{R}_{+}^{3}$,

$$
\begin{aligned}
\sup _{\left(S_{0}, \sigma, T\right) \in H} \mathbb{E}_{\mathbb{P}}\left[\left|\frac{\Phi_{k}\left(S_{T}^{(\mathrm{s})}\right)-\Phi\left(S_{T}^{(\mathrm{s})}\right)}{L_{-, T}}\right|^{p_{1}}\right] & \leq C_{H}^{p_{1}} \sup _{\left(S_{0}, \sigma, T\right) \in H} \mathbb{E}_{\mathbb{P}}\left[\left|\Phi_{k}\left(S_{T}^{(\mathrm{s})}\right)-\Phi\left(S_{T}^{(\mathrm{s})}\right)\right|^{p_{1}}\right] \\
& \leq C_{H}^{p_{1}} \sup _{\left(S_{0}, \sigma, T\right) \in H}\left\|\Phi_{k}-\Phi\right\|_{\infty}^{p_{1}} \rightarrow 0,
\end{aligned}
$$

as $k \uparrow+\infty$, where the first inequality holds by the assumption $\Phi(x)=0$ around $x_{0}$.

Step 2: Next, we extend from $C_{K}\left(\mathbb{R}_{+} ; \mathbb{R}\right)$ to $C_{b}\left(\mathbb{R}_{+} ; \mathbb{R}\right)$. Let $\Phi$ be in $C_{b}\left(\mathbb{R}_{+} ; \mathbb{R}\right)$ satisfying $\Phi(x)=0$ around $x_{0}$. Fix $\varepsilon \in(0,1)$. We can always find a sequence $\left\{\Phi_{k}\right\}_{k \in \mathbb{N}}$ of continuous functions such that

$$
\Phi_{k}(x)= \begin{cases}\Phi(x), & \text { if } x \in(0, k-\varepsilon] \\ 0, & \text { if } x \in[k+\varepsilon,+\infty)\end{cases}
$$

and for each $x \in(k-\varepsilon, k+\varepsilon), \Phi_{k}(x) \in[0, \Phi(x)]$, where an improper interval [0,-1] should be understood as [-1,0]. Clearly, for each $k \in \mathbb{N}$, the function $\Phi_{k}$ is in $C_{K}\left(\mathbb{R}_{+} ; \mathbb{R}\right)$, vanishes around $x_{0}$ and satisfies $\sup _{k \in \mathbb{N}}\left\|\Phi_{k}\right\|_{\infty}=\|\Phi\|_{\infty}$. It then holds that for each compact 
set $H \subset \mathbb{R}_{+}^{3}$,

$$
\begin{aligned}
\sup _{\left(S_{0}, \sigma, T\right) \in H} \mathbb{E}_{\mathbb{P}}\left[\left|\frac{\Phi_{k}\left(S_{T}^{(\mathrm{s})}\right)-\Phi\left(S_{T}^{(\mathrm{s})}\right)}{L_{-, T}}\right|^{p_{1}}\right] & \leq C_{H}^{p_{1}} \sup _{\left(S_{0}, \sigma, T\right) \in H} \mathbb{E}_{\mathbb{P}}\left[\left|\Phi_{k}\left(S_{T}^{(\mathrm{s})}\right)-\Phi\left(S_{T}^{(\mathrm{s})}\right)\right|^{p_{1}}\right] \\
& =C_{H}^{p_{1}} \sup _{\left(S_{0}, \sigma, T\right) \in H} \mathbb{E}_{\mathbb{P}}\left[\left|\Phi\left(S_{T}^{(\mathrm{s})}\right)\right|^{p_{1}} \mathbb{1}\left(S_{T}^{(\mathrm{s})}>k-\varepsilon\right)\right] \\
& \leq C_{H}^{p_{1}} \sup _{\left(S_{0}, \sigma, T\right) \in H} \frac{\|\Phi\|_{\infty}^{p_{1}}}{(k-\varepsilon)^{\gamma}} \mathbb{E}_{\mathbb{P}}\left[\left(S_{T}^{(\mathrm{s})}\right)^{\gamma}\right] \rightarrow 0
\end{aligned}
$$

as $k \uparrow+\infty$, where the first inequality holds by the assumption $\Phi(x)=0$ around $x_{0}$ and the last inequality holds by the Chebyshev inequality with constant $\gamma>1$.

Step 3: We next consider the class of finite linear combinations of indicator functions on an interval of $\mathbb{R}_{+}$. To this end, write $b_{ \pm}:=x_{0} \pm \delta$ for an arbitrarily small $\delta>0$ and it suffices to study the case $\Phi=\mathbb{1}_{[\tau,+\infty) \backslash\left(b_{-}, b_{+}\right)}$or $\Phi=\mathbb{1}_{(\tau,+\infty) \backslash\left(b_{-}, b_{+}\right)}$for some positive $\tau$. We can always find a sequence of $\left\{\Phi_{k}\right\}_{k \in \mathbb{N}}$ of continuous functions such that

$$
\Phi_{k}(x)= \begin{cases}\Phi(x) & \text { if } x \in[\tau+c / k,+\infty), \\ 0 & \text { if } x \in(0, \tau-c / k),\end{cases}
$$

where $c \in(0, \tau k)$, and $\Phi_{k}(x) \in[0, \Phi(x)]$ for $x \in(\tau-c / k, \tau+c / k)$. Clearly, for each $k \in \mathbb{N}$, the function $\Phi_{k}$ is in $C_{b}\left(\mathbb{R}_{+} ; \mathbb{R}\right)$, vanishes around $x_{0}$ and satisfies $\sup _{k \in \mathbb{N}}\left\|\Phi_{k}\right\|_{\infty} \leq 1$. Then, it holds that for each compact set $H \subset \mathbb{R}_{+}^{3}$,

$$
\begin{aligned}
& \sup _{\left(S_{0}, \sigma, T\right) \in H} \mathbb{E}_{\mathbb{P}}\left[\left|\frac{\Phi_{k}\left(S_{T}^{(\mathrm{s})}\right)-\Phi\left(S_{T}^{(\mathrm{s})}\right)}{L_{-, T}}\right|^{p_{1}}\right] \\
& \quad \leq C_{H}^{p_{1}} \sup _{\left(S_{0}, \sigma, T\right) \in H} \mathbb{E}_{\mathbb{P}}\left[\left|\Phi_{k}\left(S_{T}^{(\mathrm{s})}\right)-\Phi\left(S_{T}^{(\mathrm{s})}\right)\right|^{2} ; S_{T}^{(\mathrm{s})} \in\left(\tau-\frac{c}{k}, \tau+\frac{c}{k}\right)\right] \\
& \quad \leq 4 C_{H}^{p_{1}} \sup _{\left(S_{0}, \sigma, T\right) \in H} \mathbb{P}\left(S_{T}^{(\mathrm{s})} \in\left(\tau-\frac{c}{k}, \tau+\frac{c}{k}\right)\right) \\
& \leq C C_{H}^{p_{1}} \ln \frac{\tau+c / k}{\tau-c / k} \rightarrow 0,
\end{aligned}
$$

as $k \uparrow+\infty$, where the first inequality holds by the assumption $\Phi(x)=0$ around $x_{0}$, the last inequality holds by Lemma A.1(ii) and $C$ is a positive constant.

Step 4: Finally, we extend to the class of functions $\Phi=\Psi \times \mathbb{1}_{A}$, where $\Psi$ is a continuous function with linear growth and vanishes around $x_{0}$, and $A$ is an interval of $\mathbb{R}_{+}$. For a sequence $\left\{\Psi_{k}\right\}_{k \in \mathbb{N}}$ of functions in $C_{b}\left(\mathbb{R}_{+} ; \mathbb{R}\right)$ such that

$$
\Psi_{k}(x)= \begin{cases}\Psi(x) & \text { if }|\Psi(x)| \leq k \\ k & \text { if } \Psi(x)>k \\ -k & \text { if } \Psi(x)<-k\end{cases}
$$

we define $\Phi_{k}:=\Psi_{k} \times \mathbb{1}_{A}$. Clearly, $\Phi_{k} \rightarrow \Phi$ pointwise. Then, it holds that for $\gamma>1$ and for each compact set $H \subset \mathbb{R}_{+}^{3}$,

$$
\begin{aligned}
& \sup _{\left(S_{0}, \sigma, T\right) \in H} \mathbb{E}_{\mathbb{P}}\left[\left|\frac{\Phi_{k}\left(S_{T}^{(\mathrm{s})}\right)-\Phi\left(S_{T}^{(\mathrm{s})}\right)}{L_{-, T}}\right|^{p_{1}}\right] \\
& \leq C_{H}^{p_{1}} \sup _{\left(S_{0}, \sigma, T\right) \in H} \mathbb{E}_{\mathbb{P}}\left[\left|\Psi_{k}\left(S_{T}^{(\mathrm{s})}\right)-\Psi\left(S_{T}^{(\mathrm{s})}\right)\right|^{p_{1}} ;\left|\Psi\left(S_{T}^{(\mathrm{s})}\right)\right|>k\right] \\
& \leq 2^{p_{1}} C_{H}^{p_{1}} \sup _{\left(S_{0}, \sigma, T\right) \in H} \mathbb{E}_{\mathbb{P}}\left[\left|\Psi\left(S_{T}^{(\mathrm{s})}\right)\right|^{p_{1}} ;\left|\Psi\left(S_{T}^{(\mathrm{s})}\right)\right|>k\right] \\
& \leq 2^{p_{1}} k^{p_{1}(1-\gamma)} C_{H}^{p_{1}} \sup _{\left(S_{0}, \sigma, T\right) \in H} \mathbb{E}_{\mathbb{P}}\left[\left|\Psi\left(S_{T}^{(\mathrm{s})}\right)\right|^{p_{1} \gamma}\right] \\
& \leq k^{p_{1}(1-\gamma)} C C_{H}^{p_{1}} \sup _{\left(S_{0}, \sigma, T\right) \in H} \mathbb{E}_{\mathbb{P}}\left[\left(1+S_{T}^{(\mathrm{s})}\right)^{p_{1} \gamma}\right] \rightarrow 0,
\end{aligned}
$$

as $k \uparrow+\infty$, where the first inequality holds by the assumption $\Phi(x)=0$ around $x_{0}$, the third holds by the Chebyshev inequality with constant $\gamma>1$, the fourth holds by the linear growth with a positive constant $C$, and the convergence holds with the help of Lemma A.1 (iii). It is now immediate to extend to the class $\mathscr{J}\left(\mathbb{R}_{+} ; \mathbb{R}\right)$ vanishing around $x_{0}$.

The regularity conditions in the formulas (ii)-(iv) can be relaxed in a similar manner. 
Proposition A.3. It holds that

$$
\begin{aligned}
\mathbb{E}_{\mathbb{Q}_{0}}\left[\left.\frac{d \mathbb{P}}{d \mathbb{Q}_{0}}\right|_{\mathscr{G}_{1}} \Phi^{\prime}\left(\widetilde{S}_{T}^{(\mathrm{ts})}\right)\right. & \left.\widetilde{S}_{T}^{(\mathrm{ts})}\left(\eta_{-} \frac{\sigma\left(1-\alpha_{-}\right)}{\alpha_{-}} \widetilde{L}_{-, T}+\eta_{+} \frac{\sigma\left(1-\alpha_{+}\right)}{\alpha_{+}} \widetilde{L}_{+, T}\right)\right] \\
& =\mathbb{E}_{\mathbb{Q}_{0}}\left[\left.\frac{d \mathbb{P}}{d \mathbb{Q}_{0}}\right|_{\mathscr{G}_{1}} \Phi\left(\widetilde{S}_{T}^{(\mathrm{ts})}\right)\left(-\eta_{-}\left(G \frac{1-\alpha_{-}}{\alpha_{-}} \widetilde{L}_{-, T}+\left(E_{-}-1\right)\right)+\eta_{+}\left(M \frac{1-\alpha_{+}}{\alpha_{+}} \widetilde{L}_{+, T}-\left(E_{+}-1\right)\right)\right)\right]
\end{aligned}
$$

where the Radon-Nikodým derivative is defined in 3.9. In particular,

$$
\begin{aligned}
& \mathbb{E}_{\mathbb{Q}_{0}}\left[\left.\frac{d \mathbb{P}}{d \mathbb{Q}_{0}}\right|_{\mathscr{G}_{1}} \Phi^{\prime}\left(\widetilde{S}_{T}^{\mathrm{ts})}\right) \widetilde{S}_{T}^{\mathrm{ts})} \widetilde{L}_{-, T}\right]=\mathbb{E}_{\mathbb{Q}_{0}}\left[\left.\frac{d \mathbb{P}}{d \mathbb{Q}_{0}}\right|_{\mathscr{G}_{1}} \Phi\left(\widetilde{S}_{T}^{\mathrm{ts})}\right)\left(-\frac{G \widetilde{L}_{-, T}}{\sigma}-\frac{\alpha_{-}\left(E_{-}-1\right)}{\sigma\left(1-\alpha_{-}\right)}\right)\right], \\
& \mathbb{E}_{\mathbb{Q}_{0}}\left[\left.\frac{d \mathbb{P}}{d \mathbb{Q}_{0}}\right|_{\mathscr{G}_{1}} \Phi^{\prime}\left(\widetilde{S}_{T}^{\mathrm{ts})}\right) \widetilde{S}_{T}^{\mathrm{ts})} \widetilde{L}_{+, T}\right]=\mathbb{E}_{\mathbb{Q}_{0}}\left[\left.\frac{d \mathbb{P}}{d \mathbb{Q}_{0}}\right|_{\mathscr{G}_{1}} \Phi\left(\widetilde{S}_{T}^{(\mathrm{ts})}\right)\left(\frac{M \widetilde{L}_{+, T}}{\sigma}-\frac{\alpha_{+}\left(E_{+}-1\right)}{\sigma\left(1-\alpha_{+}\right)}\right)\right] .
\end{aligned}
$$

Proof. By the scaling property 2.3 , it holds that

$$
\mathbb{E}_{\mathbb{Q}_{0}}\left[\frac{e^{G \widetilde{L}_{-, T}^{\left(\eta_{-} \lambda\right)}-M \widetilde{L}_{+, T}^{\left(\eta_{+} \lambda\right)}}}{\mathbb{E}_{\mathbb{Q}_{0}}\left[e^{G L_{-, T}-M L_{+, T}}\right]} \Phi\left(\widetilde{S}_{T}^{\left(\mathrm{ts}, \eta_{-} \lambda, \eta_{+} \lambda\right)}\right)\right]=\mathbb{E}_{\mathbb{Q}_{0}}\left[\left.\frac{d \mathbb{Q}_{\lambda}}{d \mathbb{Q}_{0}}\right|_{\mathscr{G}_{3}} \frac{e^{G \widetilde{L}_{-, T}-M \widetilde{L}_{+, T}}}{\mathbb{E}_{\mathbb{Q}_{0}}\left[e^{G L_{-, T}-M L_{+, T}}\right]} \Phi\left(\widetilde{S}_{T}^{(\mathrm{ts})}\right)\right]
$$

where the Radon-Nikodým derivative is defined by (A.2). Then, by differentiating the both hand sides with respect to $\lambda$ at the origin, we get the result. The interchanges of the derivative and the expectation can be justified in a similar manner to the proof of Proposition A.2 (i). By setting $\left(\eta_{-}, \eta_{+}\right)=(1,0)$ and $\left(\eta_{-}, \eta_{+}\right)=(0,1)$, we get the rest.

Proof of Theorem 3.7 Here, we omit the justification of the interchange of derivatives and expectations to avoid overloading the proof. The Delta formula is derived by differentiating the both hand sides of

$$
\mathbb{E}_{\mathbb{Q}_{0}}\left[\frac{e^{G \widetilde{L}_{-, T}^{(\eta-\lambda)}-M \widetilde{L}_{+, T}^{\left(\eta_{+} \lambda\right)}}}{\mathbb{E}_{\mathbb{Q}_{0}}\left[e^{G L_{-, T}-M L_{+, T}}\right]} \frac{\Phi\left(\widetilde{S}_{T}^{\left(\mathrm{ts}, \eta_{-} \lambda, \eta_{+} \lambda\right)}\right)}{K_{T}^{(\lambda)}}\right]=\mathbb{E}_{\mathbb{Q}_{0}}\left[\left.\frac{d \mathbb{Q}_{\lambda}}{d \mathbb{Q}_{0}}\right|_{\mathscr{G}_{3}} \frac{e^{G \widetilde{L}_{-, T}-M \widetilde{L}_{+, T}}}{\mathbb{E}_{\mathbb{Q}_{0}}\left[e^{G L_{-, T}-M L_{+}, T}\right]} \frac{\Phi\left(\widetilde{S}_{T}^{(\mathrm{ts})}\right)}{K_{T}}\right],
$$

with respect to $\lambda$ at the origin, instead of the identity A.6. For the Gamma formula, by making use of the Delta formula, we get

$$
\begin{aligned}
\frac{\partial^{2}}{\partial S_{0}^{2}} \mathbb{E}_{\mathbb{P}}\left[\Phi\left(S_{T}^{(\mathrm{ts})}\right)\right] & =\frac{\partial}{\partial S_{0}}\left(\frac{\partial}{\partial S_{0}} \mathbb{E}_{\mathbb{P}}\left[\Phi\left(S_{T}^{(\mathrm{ts})}\right)\right]\right) \\
& =\frac{\partial}{\partial S_{0}}\left(\frac{1}{S_{0} \sigma} \mathbb{E}_{\mathbb{Q}_{0}}\left[\left.\frac{d \mathbb{P}}{d \mathbb{Q}_{0}}\right|_{\mathscr{G}_{1}} \Phi\left(\widetilde{S}_{T}^{(\mathrm{ts})}\right)\left(\frac{J_{T}}{K_{T}}+\frac{N_{T}}{K_{T}^{2}}\right)\right]\right) \\
& =\frac{1}{S_{0}^{2} \sigma} \mathbb{E}_{\mathbb{P}}\left[\left.\frac{d \mathbb{P}}{d \mathbb{Q}_{0}}\right|_{\mathscr{G}_{1}} \Phi^{\prime}\left(\widetilde{S}_{T}^{(\mathrm{ts})}\right) \widetilde{S}_{T}^{(\mathrm{ts})}\left(\frac{J_{T}}{K_{T}}+\frac{N_{T}}{K_{T}^{2}}\right)\right]-\frac{1}{S_{0}} \frac{\partial}{\partial S_{0}} \mathbb{E}_{\mathbb{P}}\left[\Phi\left(S_{T}^{(\mathrm{ts})}\right)\right] .
\end{aligned}
$$

The first term can be proceeded by differentiating the both hand sides of

$$
\begin{array}{r}
\mathbb{E}_{\mathbb{Q}_{0}}\left[\frac{e^{G \widetilde{L}_{-, T}^{\left(\eta_{-} \lambda\right)}-M \widetilde{L}_{+, T}^{\left(\eta_{+} \lambda\right)}}}{\mathbb{E}_{\mathbb{Q}_{0}}\left[e^{\left.G L_{-, T}-M L_{+, T}\right]}\right.} \Phi\left(\widetilde{S}_{T}^{\left(\mathrm{ts}, \eta_{-} \lambda, \eta_{+} \lambda\right)}\right)\left(\frac{J_{T}^{(\lambda)}}{\left(K_{T}^{(\lambda)}\right)^{2}}+\frac{N_{T}^{(\lambda)}}{\left(K_{T}^{(\lambda)}\right)^{3}}\right)\right] \\
=\mathbb{E}_{\mathbb{Q}_{0}}\left[\left.\frac{d \mathbb{Q}_{\lambda}}{d \mathbb{Q}_{0}}\right|_{\mathscr{G}_{3}} \frac{e^{G \widetilde{L}_{-, T}-M \widetilde{L}_{+, T}}}{\mathbb{E}_{\mathbb{Q}_{0}}\left[e^{\left.G L_{-, T}-M L_{+, T}\right]}\right.} \Phi\left(\widetilde{S}_{T}^{(\mathrm{s})}\right)\left(\frac{J_{T}}{K_{T}^{2}}+\frac{N_{T}}{K_{T}^{3}}\right)\right],
\end{array}
$$

with respect to $\lambda$ at the origin, where the Radon-Nikodým derivative is defined by (A.2). For the Vega formula, observe that

$$
\frac{\partial}{\partial \sigma} \mathbb{E}_{\mathbb{P}}\left[\Phi\left(S_{T}^{(\mathrm{ts})}\right)\right]=\mathbb{E}_{\mathbb{P}}\left[\Phi^{\prime}\left(S_{T}^{(\mathrm{ts})}\right) S_{T}^{(\mathrm{ts})} X_{T}^{(\mathrm{ts})}\right]-S_{0} T c_{2}^{\prime}(\sigma) \frac{\partial}{\partial S_{0}} \mathbb{E}_{\mathbb{P}}\left[\Phi\left(S_{T}^{(\mathrm{ts})}\right)\right],
$$

where the first term can be proceeded as

$$
\mathbb{E}_{\mathbb{P}}\left[\Phi^{\prime}\left(S_{T}^{(\mathrm{ts})}\right) S_{T}^{(\mathrm{ts})} X_{T}^{(\mathrm{ts})}\right]=\mathbb{E}_{\mathbb{Q}_{0}}\left[\left.\frac{d \mathbb{P}}{d \mathbb{Q}_{0}}\right|_{\mathscr{G}_{1}} \Phi^{\prime}\left(\widetilde{S}_{T}^{(\mathrm{ts})}\right) \widetilde{S}_{T}^{(\mathrm{ts})}\left(\widetilde{L}_{-, T}+\widetilde{L}_{+, T}\right)\right]+S_{0} T \gamma_{2} \frac{\partial}{\partial S_{0}} \mathbb{E}_{\mathbb{P}}\left[\Phi\left(S_{T}^{(\mathrm{ts})}\right)\right] .
$$


The rest can be done with the help of Proposition A.3 For the Theta formula,

$$
\begin{aligned}
& \frac{\partial}{\partial T} \mathbb{E}_{\mathbb{P}}\left[\Phi\left(S_{T}^{(\mathrm{ts})}\right)\right]= \frac{\partial}{\partial T} \mathbb{E}_{\mathbb{Q}_{0}}\left[\left.\frac{d \mathbb{P}}{d \mathbb{Q}_{0}}\right|_{\mathscr{G}_{1}} \Phi\left(\widetilde{S}_{T}^{(\mathrm{ts})}\right)\right] \\
&=\mathbb{E}_{\mathbb{Q}_{0}}\left[\left.\frac{d \mathbb{P}}{d \mathbb{Q}_{0}}\right|_{\mathscr{G}_{1}}\left(\frac{G \widetilde{L}_{-, T}}{\alpha_{-} T}-\frac{M \widetilde{L}_{+, T}}{\alpha_{+} T}-\gamma_{1}\right) \Phi\left(\widetilde{S}_{T}^{(\mathrm{ts})}\right)\right] \\
&+\left(r-q+\sigma \gamma_{2}-c_{2}(\sigma)\right) S_{0} \frac{\partial}{\partial S_{0}} \mathbb{E}_{\mathbb{P}}\left[\Phi\left(S_{T}^{(\mathrm{ts})}\right)\right] \\
&+\mathbb{E}_{\mathbb{Q}_{0}}\left[\left.\frac{d \mathbb{P}}{d \mathbb{Q}_{0}}\right|_{\mathscr{G}_{1}} \Phi^{\prime}\left(\widetilde{S}_{T}^{(\mathrm{ts})}\right) \widetilde{S}_{T}^{(\mathrm{ts})} \sigma\left(\frac{\widetilde{L}_{-, T}}{\alpha_{-} T}+\frac{\widetilde{L}_{+, T}}{\alpha_{+} T}\right)\right],
\end{aligned}
$$

where the rest can be done with the help of Proposition A.3

The regularity conditions on $\Phi$ can be removed in a similar manner to the proof of Theorem 3.1 since the tempered stable distribution has a $C^{\infty}$-density as well as the stable distribution by Lemma A.1. (v).

\section{References}

[1] Bally, V., Bavouzet, M. P., Messaoud, M. (2007) Integration by parts formula for locally smooth laws and applications to sensitivity computations, Annals of Applied Probability, 17(1) 33-66.

[2] Bismut, J. M. (1983) Calcul des variations stochastique et processus de sauts, Z. Wahrsch. Verw. Gebiete, 63(2) 147-235.

[3] Carr, P., Geman, H., Madan, D.B. and Yor, M. (2002) The fine structure of asset returns: An empirical investigation, J. Business, 75, $303-325$.

[4] Carr, P., Wu, L. (2003) The finite moment log stable process and option pricing, Journal of Finance, 58(2) 753-778.

[5] Chambers, J.M., Mallows, C.L., Stuck, B.W. (1976) A method for simulating stable random variables, Journal of the American Statistical Association, 71(354) 340-344.

[6] Eberlein, E., Madan, D. (2010) Short positions, rally fears and option markets, Applied Mathematical Finance, 17(1) 83-98.

[7] El-Khatib, Y., Privault, N. (2004) Computations of Greeks in a market with jumps via the Malliavin calculus, Finance and Stochastics, 8(2) 161-179.

[8] Fama, E.F. (1965) The behavior of stock market prices, Journal of Business, 38, 34-105.

[9] Fournié, E., Lasry, J. M., Lebuchoux, J., Lions, P. L., Touzi, N. (1999) Applications of Malliavin calculus to Monte Carlo methods in finance, Finance and Stochastics, 3(4) 391-412.

[10] Kawai, R., Kohatsu-Higa, A. (2010) Computation of Greeks and multidimensional density estimation for asset price models with time-changed Brownian motion, Applied Mathematical Finance, 17(4) 301-321.

[11] Kawai, R., Masuda, H. (2011) On simulation of tempered stable random variates, Journal of Computational and Applied Mathematics, 235(8) 2873-2887.

[12] Kawai, R., Takeuchi, A. (2011) Greeks formulae for an asset price model with gamma processes, Mathematical Finance, 21(4) 723-742.

[13] Kawai, R., Takeuchi, A. (2010) Sensitivity analysis for averaged asset price dynamics with gamma processes, Statistics \& Probability Letters, 80(1) $42-49$.

[14] Kawai, R. (2010) On sequential calibration for an asset price model with piecewise Lévy processes, IAENG International Journal of Applied Mathematics, 40(4) 239-246.

[15] Kawai, R., Likelihood ratio gradient estimation for Meixner distribution and Lévy processes, Computational Statistics, forthcoming, doi:10.1007/s00180-011-0288-7.

[16] Mandelbrot, B.B. (1963) The variation of certain speculative prices, Journal of Business, 36, 394-419.

[17] Rosiński, J. (2007) Tempering stable processes, Stochastic Processes and their Applications, 117(6) 677-707.

[18] Sato, K. (1999) Lévy processes and infinitely divisible distributions, Cambridge University Press.

[19] Takeuchi, A. (2010) The Bismut-Elworthy-Li type formulae for stochastic differential equations with jumps, Journal of Theoretical Probability, 23(2) 576-604.

[20] Tweedie, M.C.K. (1984) An index which distinguishes between some important exponential families, In: Statistics : Applications and New Directions: Proc. Indian Statistical Institute Golden Jubilee International Conference (eds. J. Ghosh and J. Roy) 579-604.

[21] Zolotarev, V.M. (1986) One-Dimensional Stable Distributions, American Mathematical Society, Providence, RI. 